\title{
Universal zero-bias conductance for the single-electron transistor
}

\author{
M. Yoshida \\ Departamento de Física, Instituto de Geociências e Ciências Exatas, Universidade Estadual Paulista, 13500 Rio Claro, SP, Brazil \\ A. C. Seridonio* and L. N. Oliveira \\ Departamento de Física e Informática, Instituto de Física de São Carlos, Universidade de São Paulo, 369 São Carlos, SP, Brazil
}

(Received 26 May 2009; revised manuscript received 16 November 2009; published 16 December 2009)

\begin{abstract}
The thermal dependence of the zero-bias conductance for the single electron transistor is the target of two independent renormalization-group approaches, both based on the spin-degenerate Anderson impurity model. The first approach, an analytical derivation, maps the Kondo-regime conductance onto the universal conductance function for the particle-hole symmetric model. Linear, the mapping is parametrized by the Kondo temperature and the charge in the Kondo cloud. The second approach, a numerical renormalization-group computation of the conductance as a function the temperature and applied gate voltages offers a comprehensive view of zero-bias charge transport through the device. The first approach is exact in the Kondo regime; the second, essentially exact throughout the parametric space of the model. For illustrative purposes, conductance curves resulting from the two approaches are compared.
\end{abstract}

DOI: 10.1103/PhysRevB.80.235317

PACS number(s): 73.21.La, 72.15.Qm, 73.23.Hk

\section{INTRODUCTION}

Nearly five decades ago, Anderson conceived a Hamiltonian to describe the interaction between a magnetic impurity and otherwise free conduction electrons. ${ }^{1}$ Once a daunting theoretical challenge, the Anderson Hamiltonian yielded to an essentially exact numerical diagonalization, ${ }^{2}$ followed by an exact analytical diagonalization. ${ }^{3,4}$ From these and alternative approaches, physical properties were extracted, which eased the interpretation of experimental data, ${ }^{5}$ theoretical results provided unifying views of apparently unrelated phenomena, ${ }^{6}$ quantitative comparisons brought forth novel perceptions, ${ }^{7}$ and dissections of the Anderson model brought to light the physics of nanoscale devices. ${ }^{8-12}$

The last ten years were remarkably fruitful. Parallel advances in scanning tunneling spectroscopy and in the fabrication of nanostructured semiconductor devices enhanced the interest in transport properties. ${ }^{13-25}$ In both areas, numerous experimental breakthroughs and theoretical analyses were reported, and the Anderson Hamiltonian proved spectacularly successful in more than one occasion. ${ }^{26,27}$

Notwithstanding the substantial volume of exact results, certain aspects of the model remain obscure. Consider universality, a concept important in its own right and by virtue of its diverse applications. Universal relations serve as benchmarks checking the accuracy of numerical data, as resources promoting the convergence of theoretical findings, and as instruments bridging the gap between the theorist's tablet and the laboratory logbook. The conditions under which the Anderson model exhibits universal thermodynamical properties were identified. ${ }^{2-4}$ Although one expects all properties of the model to be universal in the same domain, few firm results for the dynamical and transport properties can be found in libraries. ${ }^{28}$ The early effort of Costi et al. showed that the transport coefficients for the particle-hole symmetric Anderson model are universal. ${ }^{29}$ For asymmetric models - even ones that display universal thermodynamical properties-nonetheless, the universal curves fail to fit the numerical data, the disagreement growing with the asymmetry.
Puzzled by such contrasts, we have conducted a systematic study of the transport properties for the Anderson Hamiltonian. We combined analytical and numericalrenormalization group (NRG) tools and paid special attention to universality. In a preliminary report, ${ }^{30}$ we have discussed an Anderson model for a quantum dot side coupled to a quantum wire, a device comprising two conduction paths whose transport properties are marked by interference. ${ }^{31-35}$ Notwithstanding the constructive or destructive effects, we have been able to identify universal behavior throughout the Kondo regime, the parametrical domain favoring the formation of a magnetic moment at the quantum dot, and its progressive screening by the conduction electrons as the temperature is lowered past the scale set by the Kondo temperature $T_{K}$. Specifically, we found the thermal dependence of the conductance to map linearly onto a universal function of the temperature $T$ scaled by the Kondo temperature $T_{K}$. The mapping is itself universal; i.e., it depends on a single physical property, the ground-state phase shift $\delta$, into which the contributions from all model parameters are lumped.

This paper examines the alternative experimental setup in which a quantum dot or molecule, instead of side coupled to, is embedded in the conduction path. ${ }^{5,18,26,36-40}$ We show that the thermal dependence of the conductance maps onto the same universal function. Although linear, the mapping now depends explicitly on a model parameter-an external potential applied to the conduction electrons - and hence contrasts with the conclusion in our previous report. This dependence accounts for distinctions between the transport properties in the embedded and side-coupled arrangements. At high temperatures, for instance, potentials appropriately applied to the conduction electrons in the side-coupled geometry drive the conductance from nearly zero up to the ballistic limit $\mathcal{G}_{2}$ $=2 e^{2} / h$.

If the quantum dot is embedded in the conduction path, by contrast, the high-temperature conductance is pinned at low values and virtually insensitive to potentials applied to the conduction electrons. Our analysis shows that, in the embed- 


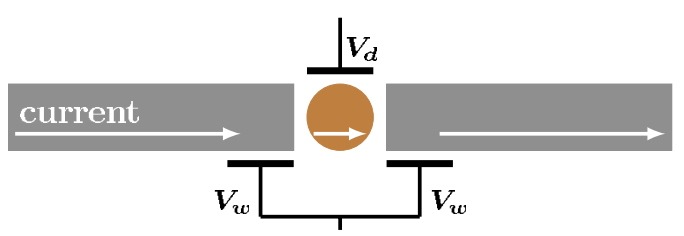

FIG. 1. (Color online) Single electron transistor. A quantum dot (circle) bridges two noninteracting quantum wires (rectangles). A gate potential $V_{d}$ controls the dot energy, while the symmetric potentials $V_{w}$ shift the energy of the wire orbitals close to the dot.

ded configuration, the dot charge parametrizes the mapping to the universal conductance curve. That charge being always close to unity in the Kondo regime, the mapping is never very far from the identity so that the conductance is always less than $25 \%$ below the universal curve.

To discuss the thermal dependence of the conductance in the Kondo regime more specifically, we have to know the Kondo temperature and the ground-state dot charge as functions of the model parameters. The Kondo regime is, moreover, only one of the physically relevant domains in the parametric space of the Anderson Hamiltonian. To offer a more comprehensive view of zero-bias conduction through the single electron transistor (SET), we have gone beyond the mapping. The second half of this paper, Secs. VI and VII, covers the Kondo range and the domains contiguous to it with a large number of temperature-dependent conductance curves. To discuss the numerical data, we take advantage of the mapping to the universal function, which links the salient features of the conductance plots to the rapid changes in dot occupation that accompany the transitions between regimes.

The transition from the Kondo regime to the neighboring domains makes the mapping to the universal function progressively less accurate. To document its decay, we also display the conductance against temperature for various gate voltages across the transition and compare the plots with the conductance curve predicted by the mapping. All illustrations considered, the numerical study provides a unified description of conduction in the single-electron transistor.

The text is divided in eight sections, more technical aspects of the analysis having been confined to the three Appendixes. Section II defines the model. Section III derives an expression relating the conductance to the spectral density of the quantum dot level. Section IV is dedicated to universality, and Sec. V to the fixed points of the model Hamiltonian and to an extension of Langreth's exact expression for the ground-state spectral density. Section VI then shows that, in the Kondo regime, the thermal dependence of the conductance can be mapped onto the symmetric-SET universal conductance.

The numerical survey is reported next. Section VII describes the numerical procedure, and Sec. VIII displays and discusses the results. Finally, Sec. IX summarizes the conclusions drawn from the analytical derivation and from the numerically computed conductances.

\section{SINGLE-ELECTRON TRANSISTOR}

Figure 1 depicts a SET, the prototypical example of em-

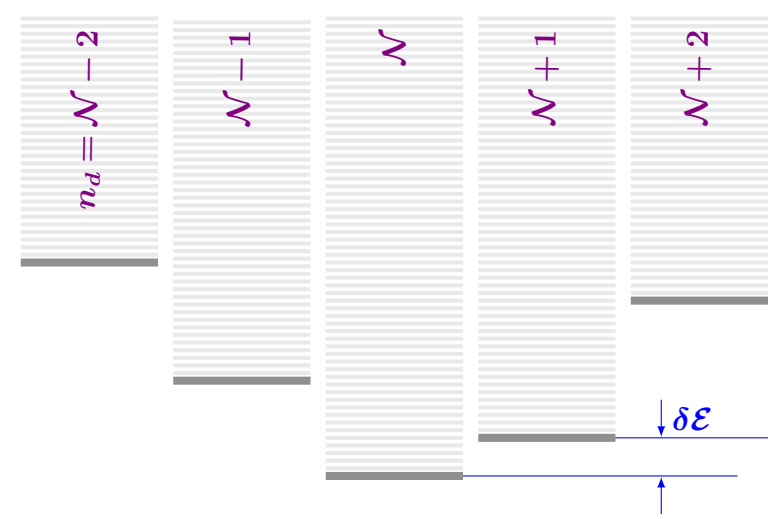

FIG. 2. (Color online) SET energies in the weak-coupling limit. The dot-level occupation $n_{d}$ labels the energies. For each $n_{d}$, the bold dash represents the conduction-band ground state, while the thinner lines represent excitations. The coupling between the dot and the two quantum wires mixes each level to the neighboring columns.

bedding. The subject of numerous experimental studies, the SET comprises two independent conduction bands coupled by a localized level. In the laboratory, the quantum dot is unevenly coupled to the electron gases represented by the two rectangles. The consequences of this asymmetry being well understood ${ }^{8-11}$ conciseness recommends that we analyze only the evenly coupled device.

Qualitatively, the physics of Fig. 1 was understood long before the first device was developed. ${ }^{8,9}$ To recapitulate, it is convenient to start from Fig. 2, which depicts the spectrum of the SET Hamiltonian $H$ in the weak-coupling limit. With the dot levels decoupled from the conduction bands, the eigenstates and eigenvalues of $H$ can be labeled by the dot quantum numbers, among which the dot occupation $n_{d}$ is chiefly important. For fixed $n_{d}$, the product of the lowest dot state by the conduction-band ground state is shown as a bold dash. The gray levels above it represent the excited states consistent with the same $n_{d}$ label.

A small transition amplitude $V$ between the quantum dot and the wires is sufficient to modify this picture. The amplitude $V$ strongly couples each gray level to the degenerate or nearly degenerate states in the neighboring columns. Exceptions are the lowest levels in the column labeled $n_{d}=\mathcal{N}$ in Fig. 2, which are energetically distant from their neighbors and thus remain unperturbed to first order in the coupling. At low temperatures, with $k_{B} T$ small in comparison with the energy $\delta \mathcal{E}$ separating the ground state from the closest level in the neighboring columns, the dot occupation is frozen at $n_{d}=\mathcal{N}$, a constraint that raises the Coulomb blockade against conduction through the dot.

Suitably adjusted, the gate potential $V_{d}$ in Fig. 1 lifts the blockade. The potential shifts the dot energies. Adjusted to the condition $\delta \mathcal{E} \approx 0$, it levels the bold dashes in the $n_{d}=\mathcal{N}$ and $n_{d}=\mathcal{N}+1$ columns in Fig. 2 so that an infinitesimal bias suffices to induce electronic flow between the wires through the dot. The zero-bias conductance peaks whenever the gate potential $V_{d}$ tunes the ground-state expectation value of $n_{d}$ to a half-integer, e.g., $\left\langle\Omega\left|n_{d}\right| \Omega\right\rangle \rightarrow \mathcal{N}+1 / 2$ as $\delta \mathcal{E} \rightarrow 0$ in Fig. 2 .

Each peak identifies a resonance at the Fermi level. As the gate voltage is swept past $\delta \mathcal{E}=0$, the ground-state occupation 
changes rapidly from $n_{d}=\mathcal{N}$ to $n_{d}=\mathcal{N}+1$, and as required by the Friedel sum rule, so does the ground-state phase shift. At moderately low temperatures, for thermal energies smaller than the average spacing between the bold dashes in the figure, the plot of the conductance against gate voltage shows a succession of peaks. Conductances measured at moderately low temperatures do display a sequence of resonances. At very low temperatures, however, the conductance pattern changes to a sequence of intervals alternating between insulating valleys and conducting plateaus.

The conducting plateaus are due to the Kondo effect. For gate voltages corresponding to odd ground-state dot occupations, the magnetic moment of the resulting dot spin interacts antiferromagnetically with the conduction electrons. As the device is cooled past the Kondo temperature, the screening of the moment creates the Kondo resonance, a spiked enhancement of the density of states anchored at the Fermi level. The pinned resonance defeats the Coulomb blockade and allows ballistic conduction through the quantum dot.

\section{ANDERSON MODEL}

A variant of the Anderson Hamiltonian encapsulates the physics of the device in Fig. 1. A spin degenerate level $c_{d}$ represents the dot level, and two structureless half-filled conduction bands, labeled $L$ (left) and $R$ (right), represent the two quantum wires. The $L(R)$ wire comprises $N$ state $c_{k L}\left(c_{k R}\right)$ with energies defined by the linear dispersion relation $\epsilon_{k}$ $=\left(k-k_{F}\right) v_{F}\left(0 \leqslant k \leqslant 2 k_{F}\right)$ so that the bandwidth is $2 D=2 v_{F} k_{F}$. The per-particle per-spin density of conduction states is $\rho$ $=1 / 2 D$, and we will let $\Delta \equiv D / N$ denote the energy splittings in the conduction bands. The model Hamiltonian is then the sum of three terms, $H=H_{w}+H_{d}+H_{w d}$, where the first term describes the wires,

$$
H_{w}=\sum_{k \alpha} \epsilon_{k} c_{k \alpha}^{\dagger} c_{k \alpha}+\frac{W}{N} \sum_{k q \alpha} c_{k \alpha}^{\dagger} c_{q \alpha},
$$

with an intrawire scattering potential $W$, fixed by the potential $V_{w}$ in Fig. 1 , and $\alpha=L, R$. The Hamiltonian $H_{d}$ describes the dot,

$$
H_{d}=\varepsilon_{d} n_{d}+U n_{d \uparrow} n_{d \downarrow},
$$

where $U$ represents the Coulomb repulsion between electrons in the dot orbital, and the dot energy $\varepsilon_{d}$ is controlled by the gate potential $V_{d}$ in Fig. 1. Finally, the Hamiltonian $H_{w d}$ couples the wires to the dot,

$$
H_{w d}=\frac{V}{\sqrt{2 N}} \sum_{k \alpha}\left(c_{k \alpha}^{\dagger} c_{d}+\text { H.c. }\right) \text {. }
$$

\section{A. Parity}

To exploit the inversion symmetry of Fig. 1, we define the normalized even $\left(a_{k}\right)$ and odd $\left(b_{k}\right)$ operators as

$$
a_{k}=\frac{1}{\sqrt{2}}\left(c_{k L}+c_{k R}\right),
$$

$$
b_{k}=\frac{1}{\sqrt{2}}\left(c_{k L}-c_{k R}\right) .
$$

The projection of the model Hamiltonian on the basis of $a_{k}$ 's and $b_{k}$ 's splits it in two decoupled pieces, $H=H_{A}+H_{B}$, where

$$
H_{A}=\sum_{k} \epsilon_{k} a_{k}^{\dagger} a_{k}+W f_{0}^{\dagger} f_{0}+V\left(f_{0}^{\dagger} c_{d}+\text { H.c. }\right)+H_{d},
$$

with the traditional NRG shorthand

$$
f_{0} \equiv \sum_{k} a_{k} / \sqrt{N}
$$

and

$$
H_{B}=\sum_{k} \epsilon_{k} b_{k}^{\dagger} b_{k}+\frac{W}{N} \sum_{k q} b_{k}^{\dagger} b_{q}
$$

\section{B. Conductance}

The odd Hamiltonian $H_{B}$ is decoupled from the quantum dot. It is, moreover, quadratic and hence easily diagonalizable. Appendix $\mathrm{C}$ determines its spectrum, analyzes the response of the conduction and dot electrons to the application of an infinitesimal bias, and turns the result into the following linear-response expression for the conductance:

$$
G(T)=\mathcal{G}_{2} \pi \Gamma_{W} \int_{-D}^{D} \rho_{d}(\epsilon, T)\left[-\frac{\partial f(\epsilon)}{\partial \epsilon}\right] d \epsilon,
$$

where $f(\epsilon)$ is the Fermi function; $\mathcal{G}_{2} \equiv 2 e^{2} / h$, the conductance quantum,

$$
\Gamma_{W}=\frac{\Gamma}{1+\pi^{2} \rho^{2} W^{2}},
$$

is the width $\Gamma=\pi \rho V^{2}$ of the $c_{d}$ level, here renormalized by the scattering potential $W$, and

$$
\rho_{d}(\epsilon, T)=\frac{1}{f(\epsilon)} \sum_{m n} \frac{e^{-\beta E_{m}}}{\mathcal{Z}}\left|\left\langle n\left|c_{d}^{\dagger}\right| m\right\rangle\right|^{2} \delta\left(\epsilon_{m n}-\epsilon\right)
$$

is the spectral density for the dot level. Here $|m\rangle$ and $|n\rangle$ are eigenstates of $H_{A}$ with eigenvalues $E_{m}$ and $E_{n}$, respectively, $\epsilon_{m n} \equiv E_{m}-E_{n}$, and $\mathcal{Z}$ is the partition function for the Hamiltonian $H_{A}$.

As one would expect, given that the odd Hamiltonian $H_{B}$ commutes with $c_{d}$, only the eigenvalues and eigenvectors of $H_{A}$ are needed to compute the right-hand sides of Eqs. (8) and (10). The following discussion will hence focus on the even Hamiltonian, Eq. (5), which is equivalent to the conventional spin-degenerate Anderson Hamiltonian. ${ }^{1}$

\section{Characteristic energies}

Four characteristic energies govern the physical properties of the Anderson Hamiltonian. Two of them are the chargeexcitation energies catching the eye in Fig. 3: the energy $-\epsilon_{d}$ needed to remove an electron from the dot level and the energy $\epsilon_{d}+U$ needed to add an electron to the level. The 


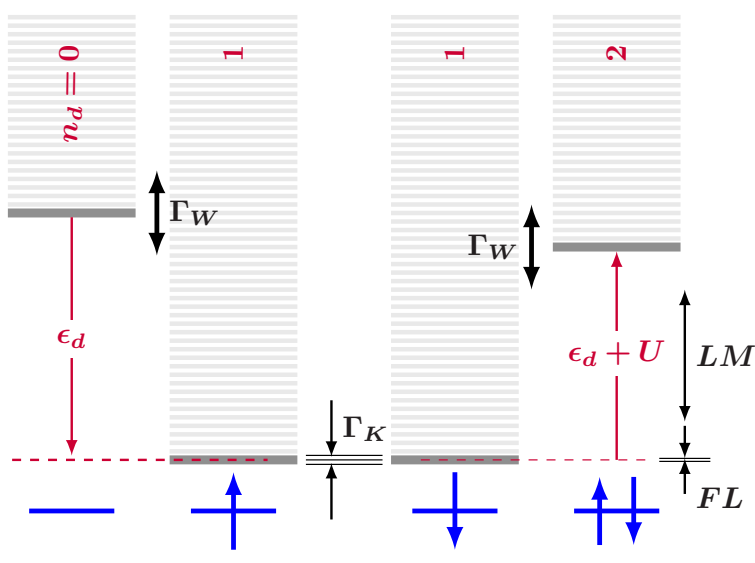

FIG. 3. (Color online) Spectrum of the spin-degenerate Anderson model, displayed as in Fig. 2. In the weak-coupling limit, the eigenstates are labeled by the occupation $n_{d}$ and spin component of the dot configuration displayed at the bottom. For $V \neq 0$, each level in the left and right columns hybridizes with nearly degenerate levels in the central columns and acquires the width $\Gamma_{W}$ in Eq. (9). At low energies, the levels in the two central columns combine into a singlet and acquire a width $\Gamma_{K} \sim k_{B} T_{K}$. The vertical arrows near the right border mark the domains of the LM and FL fixed points.

particle-hole transformation $c_{k} \rightarrow c_{k}^{\dagger}, c_{d} \rightarrow-c_{d}^{\dagger}$ swaps the two energies so that the transformed dot Hamiltonian is given by the right-hand side of Eq. (2) with $\epsilon_{d} \rightarrow-\left(\epsilon_{d}+U\right)$.

If $2 \epsilon_{d}+U=0$, the dot Hamiltonian remains invariant under the particle-hole transformation. If, in addition, $W=0$, Eq. (5) reduces to the symmetric Hamiltonian

$$
H_{A}^{S}=\sum_{k} \epsilon_{k} a_{k}^{\dagger} a_{k}+V\left(f_{0}^{\dagger} c_{d}+\text { H.c. }\right)-\frac{U}{2}\left(n_{d \uparrow}-n_{d \downarrow}\right)^{2} .
$$

With $V \neq 0$, two other energies arise: the level width $\Gamma_{W}[\mathrm{Eq}$. (9)] and the Kondo energy $k_{B} T_{K}$, given by

$$
T_{K} \sim \sqrt{\rho J} \exp (-1 / \rho J),
$$

where $J$ is the antiferromagnetic interaction between the conduction electrons and the dot magnetic moment, ${ }^{41}$

$$
\rho J=2 \frac{\Gamma_{W}}{\pi\left|\varepsilon_{d}\right|} \frac{U}{\varepsilon_{d}+U} .
$$

In the Kondo regime, thermal and excitation energies are much smaller than $\min \left(\left|\varepsilon_{d}\right|, \varepsilon_{d}+U\right)$. In Fig. 3, only the lowest levels in the central columns are energetically accessible. The energy $\Gamma_{W}$, associated with transitions from the central to the external columns in the figure (i.e., with $c_{d}^{1} \rightarrow c_{d}^{2}$ and $c_{d}^{1} \rightarrow c_{d}^{0}$ transitions) becomes inoperant. Instead, at very low excitation and thermal energies, smaller than the Kondo energy $k_{B} T_{K}$, the dot spin binds antiferromagnetically to the conduction spins. In Fig. 3, the lowest states in the left and right central columns hybridize to constitute a Kondo singlet.

\section{UNIVERSALITY}

The concepts recapitulated in Sec. III C emerged over three decades ago, with the first accurate computation of the

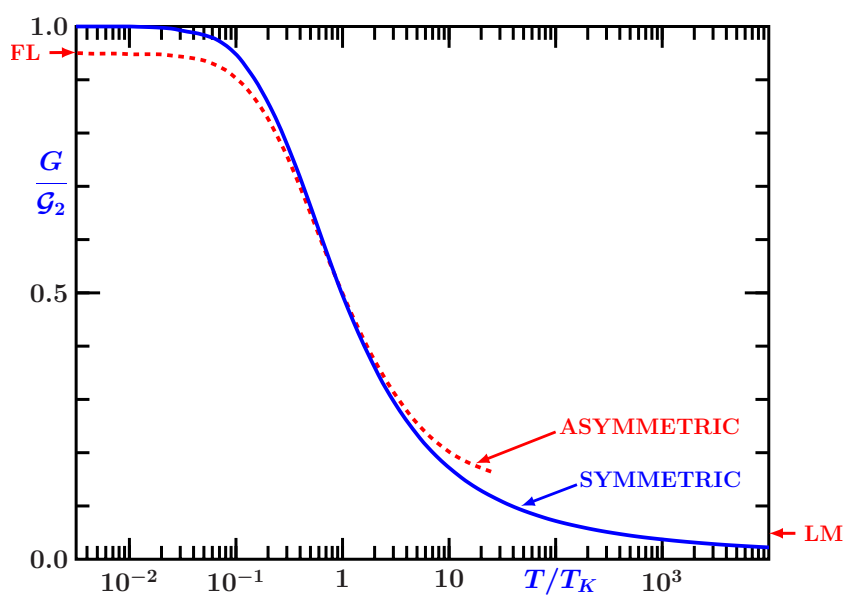

FIG. 4. (Color online) Thermal dependences of the conductance for two sets of model parameters, computed by the procedure in Sec. VII. The solid line depicts the universal conductance curve (Ref. 29) for the symmetric Hamiltonian (11). Here, it was computed with $\Gamma=0.1 D$ and $U=3 D$. The temperatures were scaled by the Kondo temperature $T_{K}=2.4 \times 10^{-6} \mathrm{D} / k_{B}$, fixed by the requirement $G\left(T_{K}\right)=0.5 \mathcal{G}_{2}$. The dashed curve is the conductance for Hamiltonian (5) with $\Gamma=0.1 D, U=3 D, \varepsilon_{d}=-0.3 D$, and $W=0$, which yielded $T_{K}=4.0 \times 10^{-3} D$. To keep the data within the temperature range $k_{B} T<0.1 D$, the dashed plot stops at $T=25 T_{K}$. The horizontal arrows pointing to the vertical axes indicate the corresponding fixed-point conductances, given by Eqs. (22a) and (22b).

magnetic susceptibility of the Anderson model, ${ }^{2}$ long before the first essentially exact computation of the conductance. A particularly important result in the more recent survey of transport properties of Costi et al. ${ }^{29}$ is the thermal dependence of the conductance for the symmetric Hamiltonian $H_{A}^{S}$, the universal curve $G^{S}\left(T / T_{K}\right)$, depicted by the solid line in Fig. 4. For $k_{B} T \ll D$ and any pair $(\Gamma, U)$ satisfying $\Gamma \ll U$ in Eq. (11), proper adjustment of the Kondo temperature $T_{K}$ gives a conductance curve $G\left(T / T_{K}\right)$ that reproduces $G^{S}\left(T / T_{K}\right)$.

In Fig. 4, for instance, the solid line was computed from the eigenvalues and eigenvectors of $H_{A}^{S}$ with $\Gamma=0.1 D$ and $U=3 D$. The definition $G\left(T_{K}\right) \equiv 0.5 \mathcal{G}_{2}$ yielded the Kondo temperature $T_{K}=2.4 \times 10^{-6} \mathrm{D}$. When the calculation was repeated for $U=0.6 D$ and the same $\Gamma$, the Kondo temperature grew four orders of magnitude to $T_{K}=2.2 \times 10^{-2} D$. Still, for $k_{B} T<0.1 D$, the plot of $G\left(T / T_{K}\right)$ resulted indistinguishable from the solid curve. While $T_{K}$ is model-parameter dependent, $G\left(T / T_{K}\right)$ is not.

Particle-hole asymmetry drives $G$ away from $G^{S}$. For $U$ $+2 \epsilon_{d} \neq 0$ or $W \neq 0$, the universal curve $G^{S}\left(T / T_{K}\right)$ no longer matches $G\left(T / T_{K}\right)$. An example is the dashed curve in Fig. 4, calculated with $\Gamma=0.1 D, U=3 D, \varepsilon_{d}=-0.3 D$, and $W=0$. The definition $G\left(T_{K}\right)=0.5 \mathcal{G}_{2}$, which in this case yields $T_{K}=4$ $\times 10^{-3} \mathrm{D}$, forces the solid and the dashed lines to agree at $T=T_{K}$; the conductance for the asymmetric model nonetheless undershoots (overshoots) the universal curve for $T$ $<T_{K}\left(T>T_{K}\right)$. To reconcile this discrepancy with the concept of universality, the following sections rely on renormalization-group concepts. 


\section{FIXED POINTS}

Renormalization-group theory probes the spectrum of Hamiltonians in search of characteristic energies and scaling invariances. The wire Hamiltonian (1), for instance, exhibits a single trivial characteristic energy: the conduction bandwidth $2 D$. For energies $\epsilon \ll D$, therefore, its spectrum is invariant under the scaling transformation $H_{w} \rightarrow \Lambda H_{w}$, for arbitrary scaling parameter $\Lambda>1$. Accordingly, for $\epsilon \ll D$, the wire Hamiltonian is a stable fixed point of the renormalization-group transformation in Ref. 2.

Latent in the Anderson Hamiltonian (5), by contrast, are the four nontrivial characteristic energies discussed in Sec. III C. Part of the spectrum of $H_{A}$ lies close to fixed points; the remainder is in transition ranges. In the vicinity of a fixed point, the spectrum remains approximately invariant under scaling; in the transition intervals, the eigenvalues are comparable to one or more characteristic energies and hence change rapidly under scale transformations. In particular, the portion of the spectrum pertinent to the Kondo regime comprises two lines of fixed points and a crossover region.

For given thermal or excitation energy $\mathcal{E}$, the inequality $\max \left(\mathcal{E}, \Gamma_{W}\right) \ll \min \left(\left|\varepsilon_{d}\right|, \varepsilon_{d}+U, D\right)$ defines the Kondo regime. As Fig. 3 shows, the dot occupation is then nearly unitary. In the energy range $k_{B} T_{K} \ll \mathcal{E} \ll \min \left(\left|\varepsilon_{d}\right|, \varepsilon_{d}+U, D\right)$, the Hamiltonian $H_{A}$ is near the local moment (LM) fixed point. At very low energies, $\mathcal{E} \ll k_{B} T_{K}$, i.e., below the energy scale defined by the narrow set of levels at the center of Fig. 3, the spectrum becomes asymptotically invariant under scaling as the Hamiltonian approaches the frozen level (FL) fixed-point. In the intermediate region $\mathcal{E} \sim k_{B} T_{K}$, the Hamiltonian crosses over from the LM to the FL.

\section{A. Fixed-point Hamiltonians}

As the two central columns in Fig. 3 indicate, the LM is an unstable fixed-point consistent of a conduction band and a free spin-1/2 variable. In the FL, a singlet replaces the spin, and the Hamiltonian is equivalent to a conduction band-a stable fixed point. In their most general form, the fixed-point conduction bands mimic the wire Hamiltonian, i.e.,

$$
H_{L M}^{*}=\sum_{k} \epsilon_{k} a_{k}^{\dagger} a_{k}+W_{L M} f_{0}^{\dagger} f_{0},
$$

and

$$
H_{F L}^{*}=\sum_{k} \epsilon_{k} a_{k}^{\dagger} a_{k}+W_{F L} f_{0}^{\dagger} f_{0},
$$

with scattering potentials $W_{F L}$ and $W_{L M}$ dependent on $V, W$, $U$, and $\epsilon_{d}$. Equations (14) and (15) identify two lines of fixed points, parametrized by $W_{L M}$ and $W_{F L}$, respectively.

The Schrieffer-Wolff transformation offers an approximation for the LM potential,

$$
\rho W_{L M}=\rho W+2 \frac{\Gamma_{W}}{\pi\left|\varepsilon_{d}\right|} \frac{2 \varepsilon_{d}+U}{\varepsilon_{d}+U} .
$$

For most applications, this expression is insufficiently accurate, and an NRG computation is necessary to determine $W_{L M}$ and $W_{F L}$. The exception is Hamiltonian (11), for which
$W_{L M}=0$, as required by particle-hole symmetry.

\section{B. Fixed-point phase shifts}

Appendix A diagonalizes the quadratic Hamiltonians (14) and (15). For the LM, the diagonal form reads

$$
H_{L M}^{*}=\sum_{k} \varepsilon_{\ell} g_{\ell}^{\dagger} g_{\ell},
$$

with phase-shifted energies

$$
\varepsilon_{\ell}=\epsilon_{\ell}-\frac{\delta_{L M}}{\pi} \Delta .
$$

Here $\Delta$ is the energy splitting defined in Sec. III.

At the LM, all conduction states are uniformly phase shifted, with

$$
\tan \delta_{L M}=-\pi \rho W_{L M} .
$$

For $H_{A}=H_{A}^{S}$, in particular, $\delta_{L M}=0$, and the low-energy eigenvalues $\varepsilon_{k}$ coincide with the $\epsilon_{k}$.

The FL eigenvalues are likewise uniformly phase shifted,

$$
H_{F L}^{*}=\sum_{k} \widetilde{\varepsilon}_{k} \widetilde{g}_{k}^{\dagger} \widetilde{g}_{k},
$$

where $\widetilde{\varepsilon}_{k}=\epsilon_{k}-(\delta / \pi) \Delta$. From the Friedel sum rule, it follows that $^{42}$

$$
\delta=\delta_{L M}+\frac{\pi}{2} .
$$

For $H_{A}=H_{A}^{S}$, in particular, $\delta=\pi / 2$.

\section{Conductance at the fixed points}

The LM is the fixed point to which the Anderson Hamiltonian would come if $\Gamma=0$, i.e., if the dot were decoupled from the electron gases. For $0<\Gamma \ll \min \left(\left|\epsilon_{d}\right|, \epsilon_{d}+U, D\right)$, although the renormalization-group flow never reaches the LM, it brings $H_{A}$ close to the fixed point. The substantial portion of the spectrum of $H_{A}$ marked by the thin doubleheaded arrow in Fig. 3 is approximately described by the many-body eigenvalues of $H_{L M}^{*}$, and in the pertinent energy range, the physical properties of $H_{A}$ and $H_{L M}^{*}$ are approximately the same. Likewise, at low temperatures, the properties of $H_{A}$ approach those of $H_{F L}^{*}$.

The renormalization-group flow of the Hamiltonian controls the thermal dependence of its physical properties. The electrical conductance is no exception. As the temperature is reduced from $T \gg T_{K}$ to $T \ll T_{K}$, as illustrated by the curves in Fig. 4, the conductance crosses over from a lower plateau to a higher one. The extension of Langreth's expression ${ }^{42}$ derived in Appendix B determines the plateau conductances,

$$
\begin{gathered}
G_{L M}=\mathcal{G}_{2} \sin ^{2}\left(\delta_{L M}-\delta_{W}\right)=\mathcal{G}_{2} \cos ^{2}\left(\delta-\delta_{W}\right), \\
G_{F L}=\mathcal{G}_{2} \sin ^{2}\left(\delta-\delta_{W}\right),
\end{gathered}
$$

where $\delta_{W}$ is the ground-state phase shift for $V=0$. According to the analysis in Appendix A, 


$$
\tan \delta_{W}=-\pi \rho W
$$

The solid curve in Fig. 4 was computed for $H_{A}=H_{A}^{S}$ so that $\delta_{w}=0$, while the ground-state (i.e., FL) phase shift is $\delta$ $=\pi / 2$. The high-temperature (low-temperature) tendency of the plot, $G\left(T \gg T_{K}\right) \rightarrow 0\left[G\left(T \ll T_{K}\right) \rightarrow \mathcal{G}_{2}\right]$, agrees with Eq. (22a) [Eq. (22b)]. The dashed curve was also computed with $\delta_{w}=0$, but the ground-state phase shift is smaller than $\pi / 2$ because the Hamiltonian now lacks particle-hole symmetry. From the low-energy eigenvalues in the NRG run that generated the conductance curve we find $\delta=0.43 \pi$. The conductances predicted by Eqs. (22) are indicated by the two horizontal arrows in Fig. 4.

Since the width $\Gamma=0.1 D$ and the dot energy $\left|\varepsilon_{d}\right|=0.3 D$ fail to satisfy the condition $\Gamma \ll\left|\varepsilon_{d}\right|$, the renormalizationgroup flow of the asymmetric Hamiltonian in Fig. 4 bypasses the LM. The Kondo thermal energy for the asymmetric Hamiltonian is $k_{B} T_{K}=4 \times 10^{-3} \mathrm{D}$. This relatively high energy leaves no room in the renormalization-group path for the thermal bracket $D \gg k_{B} T \gg k_{B} T_{K}$, which defines the LM. Instead of crossing from the LM to the FL, the Hamiltonian therefore crosses over from the band-edge regime $k_{B} T \approx D$ to the FL, Kondo screening takes place before the dot moment is fully formed, and even at the highest temperatures in Fig. 4 , the conductance is well above the horizontal arrow pointing to $G_{L M}$. At low temperatures, by contrast, the renormalization-group flow drives $H_{A}$ toward $H_{F L}^{*}$, and the dashed curve in Fig. 4 rises to $G_{F L}$.

\section{CROSSOVER}

In the Kondo regime, the Schrieffer-Wolff transformation ${ }^{41}$ brings the Anderson Hamiltonian $H_{A}$ to the Kondo form

$$
H_{J}=\sum_{k} \epsilon_{k} a_{k}^{\dagger} a_{k}+W_{L M} f_{0}^{\dagger} f_{0}+J \sum_{\mu \nu} f_{0 \mu}^{\dagger} \boldsymbol{\sigma}_{\mu \nu} f_{0 \nu} \cdot \boldsymbol{S},
$$

with $J$ defined in Eq. (13).

To eliminate the scattering potential on the right-hand side, it is convenient to project $H_{J}$ upon the basis of the eigenoperators $g_{k}$ of the LM, which yields ${ }^{43}$

$$
H_{J}=\sum_{k} \varepsilon_{\ell} g_{\ell}^{\dagger} g_{\ell}+J_{W} \sum_{\mu \nu} \phi_{0 \mu}^{\dagger} \boldsymbol{\sigma}_{\mu \nu} \phi_{0 \nu} \cdot S,
$$

where $J_{W}=J \cos ^{2} \delta_{L M}$, and

$$
\phi_{0}=\frac{1}{\sqrt{N}} \sum_{\ell} g_{\ell} .
$$

In the symmetric case $\delta_{L M}$ vanishes, and the operator $\phi_{0}$ reduces to $f_{0}$.

In the Kondo regime, the second term on the right-hand side of Eq. (25) drives the Hamiltonian from the LM to the FL. Along the resulting trajectory, the eigenvalues of $H_{J}$ scale with $T_{K} \cdot{ }^{3,4,44,45}$ Let $T_{K}$ and $\bar{T}_{K}<T_{K}$ be the Kondo temperatures corresponding to two sets of model parameters in the Kondo regime: $\mathcal{M} \equiv\left\{\Gamma, W, U, \varepsilon_{d}\right\}$ and $\overline{\mathcal{M}}$ $\equiv\left\{\bar{\Gamma}, \bar{W}, \bar{U}, \bar{\varepsilon}_{d}\right\}$, to which correspond the antiferromagnetic couplings $J$ and $\bar{J}$, respectively. If $|m\rangle$ is an eigenvector of $H_{J}$ with eigenvalue $E_{m}$, then a corresponding eigenvector $|\bar{m}\rangle$ of $H_{J}^{-}$, the scaling image of $|m\rangle$, can always be found, with the same quantum numbers and eigenvalue $\bar{E}_{m}$ such that $E_{m} / T_{K}=\bar{E}_{m} / \bar{T}_{K}$

The matrix elements of any linear combination of the operators $g_{k}$ are moreover universal. Given two eigenstates $|m\rangle$ and $|n\rangle$ of $H_{J}$ and their scaling images $|\bar{m}\rangle$ and $|\bar{n}\rangle$, then the matrix elements of $\phi_{0}$, for example, are equal: $\left\langle m\left|\phi_{0}\right| n\right\rangle$ $=\left\langle\bar{m}\left|\phi_{0}\right| \bar{n}\right\rangle$. Likewise, the matrix elements of the normalized operator

$$
\phi_{1}=\sqrt{\frac{3}{N}} \sum_{\ell} \frac{\varepsilon_{\ell}}{D} g_{\ell}
$$

are universal: $\left\langle m\left|\phi_{1}\right| n\right\rangle=\left\langle\bar{m}\left|\phi_{1}\right| \bar{n}\right\rangle$.

\section{A. Thermal dependence of the conductance}

By contrast, the matrix elements $\left\langle m\left|c_{d}\right| n\right\rangle$ on the righthand side of Eq. (10) are nonuniversal. Even at the lowest energies, as Eq. (B14) shows, they depend explicitly on the model parameters. To discuss universal properties, therefore, we must relate them to universal matrix elements, such as $\left\langle m\left|\phi_{0}\right| n\right\rangle,\left\langle m\left|\phi_{1}\right| n\right\rangle$, or $\left\langle m\left|g_{\ell}\right| n\right\rangle$. As a first step toward that goal, we evaluate the commutator

$$
\left[H_{A}, a_{q}^{\dagger}\right]=\epsilon_{q} a_{q}^{\dagger}+\frac{V}{\sqrt{N}} c_{d}^{\dagger}+\frac{W}{N} \sum_{p} a_{p}^{\dagger},
$$

and sum the result over $q$ to find that

$$
\left[H_{A}, f_{0}^{\dagger}\right]=\frac{1}{\sqrt{3}} f_{1}^{\dagger}+V c_{d}^{\dagger}+W f_{0}^{\dagger} .
$$

Here we have defined another shorthand

$$
f_{1}=\sqrt{\frac{3}{N}} \sum_{q} \frac{\epsilon_{q}}{D} a_{q} .
$$

Equation (29) relates the matrix elements of $c_{d}^{\dagger}$ between two (low-energy) eigenstates $|m\rangle$ and $|n\rangle$ of $H_{A}$ to those of the operators $f_{0}$ and $f_{1}$,

$$
V\left\langle m\left|c_{d}^{\dagger}\right| n\right\rangle=\left(E_{m}-E_{n}-W\right)\left\langle m\left|f_{0}^{\dagger}\right| n\right\rangle-\sqrt{3} D\left\langle m\left|f_{1}^{\dagger}\right| n\right\rangle .
$$

In the Kondo regime, with $\max \left(E_{m}, E_{n}\right) \ll D$, the first two terms within the parentheses on the right-hand side can be dropped.

In the symmetric case, since $f_{0}\left(f_{1}\right)$ coincides with $\phi_{0}\left(\phi_{1}\right)$, Eq. (31) shows that the product $V\left\langle m\left|c_{d}\right| n\right\rangle$ is universal, in line with the firmly established notion that $\Gamma \rho_{d}\left(\epsilon / k_{B} T_{K}, T / T_{K}\right)$ and $G^{S}\left(T / T_{K}\right)$ are universal functions. ${ }^{29,28}$ To discuss asymmetric Hamiltonians, we have to relate the operators $f_{0}$ and $f_{1}$ to $\phi_{0}$ and $\phi_{1}$. This is done in Appendix A 2, which shows that, in the Kondo regime, a linear transformation with model-parameter dependent coefficients relates the matrix elements of both $f_{0}$ and $f_{1}$ to those of $\phi_{0}$ and $\phi_{1}$. When Eq. (A21) is substituted for $f_{0}$ and $f_{1}$ on the right-side of Eq. (31), it results that 


$$
\sqrt{\pi \rho \Gamma_{W}}\left\langle m\left|c_{d}^{\dagger}\right| n\right\rangle=\alpha_{0}\left\langle m\left|\phi_{0}^{\dagger}\right| n\right\rangle+\alpha_{1}\left\langle m\left|\phi_{1}^{\dagger}\right| n\right\rangle .
$$

Here, the constants $\alpha_{0}$ and $\alpha_{1}$ are combinations of the (unknown) linear coefficients on the right-hand side of Eq. (A21), the parameter $W$ on the right-hand side of Eq. (31), and the ratio $\sqrt{\pi \rho \Gamma_{W}} / V$, by which we multiplied Eq. (31) to shorten the following algebra.

Substitution in Eq. (10) yields an expression relating the spectral density $\rho_{d}$ to universal functions,

$$
\pi \rho \Gamma_{W} \rho_{d}(\epsilon, T)=\alpha_{0}^{2} \rho_{0}(\epsilon, T)+\alpha_{1}^{2} \rho_{1}(\epsilon, T)+\alpha_{0} \alpha_{1} \rho_{(01)}(\epsilon, T),
$$

where

$$
\rho_{j}(\epsilon, T)=\sum_{m n} \frac{e^{-\beta E_{m}}}{\mathcal{Z} f(\epsilon)}\left|\left\langle n\left|\phi_{j}\right| m\right\rangle\right|^{2} \delta\left(E_{m}-E_{n}-\epsilon\right) \quad(j=0,1),
$$

and

$$
\begin{aligned}
\rho_{(01)}(\epsilon, T)= & \sum_{m n} \frac{e^{-\beta E_{m}}}{\mathcal{Z} f(\epsilon)}\left(\left\langle m\left|\phi_{0}^{\dagger}\right| n\right\rangle\left\langle n\left|\phi_{1}\right| m\right\rangle+\text { c.c. }\right) \\
& \times \delta\left(E_{m}-E_{n}-\epsilon\right) .
\end{aligned}
$$

Next, we substitute Eq. (33) on the right-hand side of Eq. (8) to split the conduction into three pieces,

$$
G(T)=\alpha_{0}^{2} G_{0}(T)+\alpha_{1}^{2} G_{1}(T)+\alpha_{0} \alpha_{1} G_{(01)}(T),
$$

where

$$
G_{j}(T)=\frac{\mathcal{G}_{2}}{\rho} \int_{-D}^{D} \rho_{j}(\epsilon, T)\left[-\frac{\partial f(\epsilon)}{\partial \epsilon}\right] d \epsilon \quad(j=0,1),
$$

and

$$
G_{(01)}(T)=\frac{\mathcal{G}_{2}}{\rho} \int_{-D}^{D} \rho_{(01)}(\epsilon, T)\left[-\frac{\partial f(\epsilon)}{\partial \epsilon}\right] d \epsilon .
$$

\section{B. Universal contributions to the conductance}

Given the universality of the energies $E_{m}$ and of the matrix elements $\left\langle m\left|\phi_{j}\right| n\right\rangle(j=1,2)$ on the right-hand sides of Eqs. (34) and (35), we see that the spectral densities $\rho_{j}(\epsilon, T)(j=0,1)$ and $\rho_{(01)}(\epsilon, T)$ are universal. Inspection of the right-hand sides of Eqs. (37) and (38) shows that the functions $G_{j}(j=0,1)$ and $G_{(01)}$ are likewise universal. To compute them, we are free to consider any convenient Kondo-regime Hamiltonian.

Particle-hole symmetry makes $H_{A}^{S}$ especially convenient. To show that the cross terms make no contribution to the conductance, i.e., that $G_{(01)}(T)=0$, we only have to notice that, while leaving $H_{A}^{S}$ unchanged, the particle-hole transformation $c_{d} \rightarrow-c_{d}^{\dagger}, g_{k} \rightarrow g_{k}^{\dagger}$ (i.e., $a_{k} \rightarrow a_{k}^{\dagger}$ ) reverses the sign of the product of matrix elements $\left\langle m\left|\phi_{i}^{\dagger}\right| n\right\rangle\left\langle n\left|\phi_{j}\right| m\right\rangle+$ c.c. on the right-hand side of Eq. (35). We see that $\rho^{(01)}(\epsilon, T)$ is an odd function of $\epsilon$ so that the integral on the right-hand side of Eq. (38) vanishes.

To evaluate $G_{0}$ and $G_{1}$, we start out from the closed form resulting from the diagrammatic expansion (in the coupling
$V)$ of the conduction-electron retarded Green's function for the symmetric Hamiltonian,

$$
\mathbb{G}_{k k^{\prime}}^{S}(\epsilon)=\mathbb{G}_{k}^{(0)}(\epsilon) \delta_{k k^{\prime}}+\frac{V^{2}}{N} G_{k}^{(0)}(\epsilon) \mathbb{G}_{d}^{S}(\epsilon) G_{k^{\prime}}^{(0)},
$$

where $\mathrm{G}_{d}^{S}$ is the retarded dot-level Green's function for the symmetric Hamiltonian, and

$$
\mathrm{G}_{k}^{(0)}(\epsilon)=\frac{1}{\epsilon-\epsilon_{k}+i \eta}
$$

is the free conduction-electron retarded Green's function.

From $G_{k k^{\prime}}^{S}$, it is a simple matter to obtain the spectral densities on the right-hand side of Eq. (33),

$$
\rho_{0}(\epsilon, T)=-\frac{1}{\pi N} \mathfrak{J} \sum_{k k^{\prime}} \mathbb{G}_{k k^{\prime}}^{S}(\epsilon),
$$

and

$$
\rho_{1}(\epsilon, T)=-\frac{3}{\pi N D^{2}} \mathfrak{J} \sum_{k k^{\prime}} \epsilon_{k} \epsilon_{k^{\prime}} \mathrm{G}_{k k^{\prime}}^{S}(\epsilon) .
$$

To compute the conductances at temperatures $T$ satisfying $k_{B} T \ll D$, we only need the spectral densities for $\epsilon \ll D$. It is an excellent approximation, therefore, to expand the righthand side of Eq. (40) to linear order in $\epsilon / D$,

$$
\mathrm{G}_{k}^{(0)}(\epsilon)=\frac{2 \epsilon}{D}-i \pi \delta\left(\epsilon-\epsilon_{k}\right) \quad(\epsilon \ll D) .
$$

The sums over momenta on the right-hand side of Eqs. (41) and (42) are then easily computed. Among the resulting terms, only the even powers of $\varepsilon$ contribute to the integral on the right-hand side of Eq. (37). To compute the conductance to $\mathcal{O}\left[\left(k_{B} T / D\right)^{2}\right]$ we hence neglect the terms of $\mathcal{O}(\epsilon / D)$. Equation (41) then gives

$$
\rho_{0}(\epsilon, T)=\rho-\pi \rho \Gamma \rho_{d}^{S}(\epsilon, T) .
$$

Substitution on the right-hand side of Eq. (37) then shows that the universal function $G_{0}$ is related to the universal conduction for the symmetric Hamiltonian,

$$
G_{0}(T)=\mathcal{G}_{2}-G^{S}(T) .
$$

Equation (45) becomes exact, asymptotically, at low temperatures. In the Kondo regime, the deviations, of $\mathcal{O}\left[\left(k_{B} T / D\right)^{2}\right]$, are insignificant. As an illustration, the open circles in Fig. 5 show NRG data for the conductance $G_{0}(T)$, Eq. (37), in excellent agreement with the solid line representing the right-hand side of Eq. (45).

To the same accuracy, we can neglect the $\mathcal{O}(\epsilon / D)$ terms resulting from the summation on the right-hand side of Eq. (42), which yields

$$
\rho_{1}(\epsilon, T)=\frac{6 \rho \Gamma}{\pi} \rho_{d}(\epsilon, T) .
$$

Equation (37) then shows that $G_{1}$ is also related to the conductance for the symmetric Hamiltonian, 


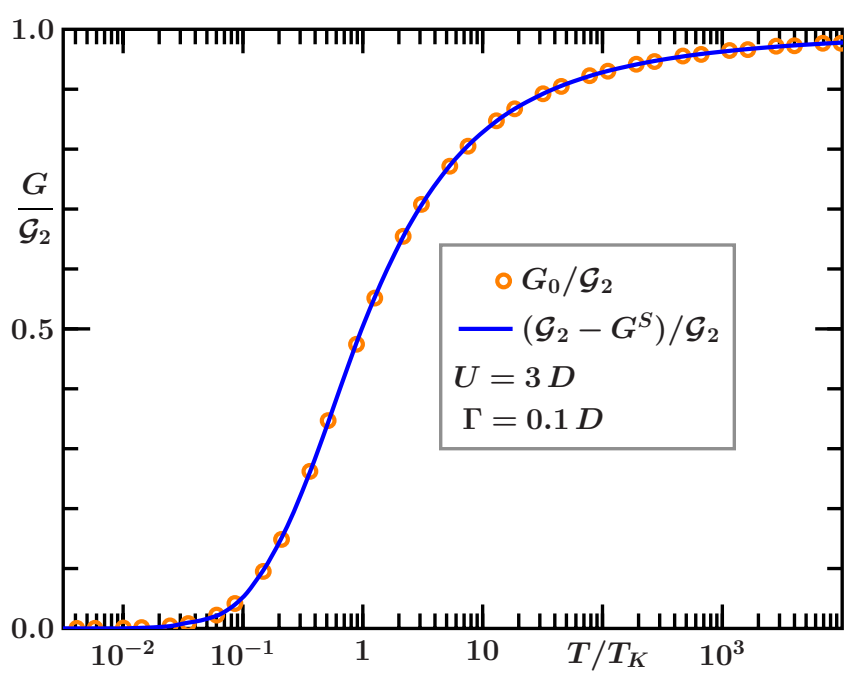

FIG. 5. (Color online) NRG results for the thermal dependence of the auxiliary conductance $G_{0}(T)$, associated with the spectral density for the operator $\phi_{0}$. The open circles show Eq. (37) for $j$ $=0$, computed for the symmetric Hamiltonian with the displayed model parameters. The solid line is the right-hand side of Eq. (45), i.e., the universal curve in Fig. 4 subtracted from the quantum conductance $\mathcal{G}_{2}$.

$$
G_{1}(T)=\frac{6}{\pi^{2}} G^{S}(T)
$$

\section{Mapping to the universal conductance}

We next combine Eqs. (45) and (47) with the result $G_{(01)}=0$ to reduce Eq. (36) to the equality

$$
G(T)=\alpha_{0}^{2}\left(\mathcal{G}_{2}-G^{S}(T)\right)+\alpha_{1}^{2} \frac{6}{\pi^{2}} G^{S}(T) .
$$

To determine the coefficients $\alpha_{0}$ and $\alpha_{1}$, we need only compare the right-hand side with the fixed-point expressions for the conductance. At the LM, $G^{S}=0$, and Eq. (22a) shows that $\alpha_{0}^{2}=\cos ^{2}\left(\delta-\delta_{W}\right)$. At the FL, $G^{S}=\mathcal{G}_{2}$, and Eq. (22b) shows that $\left(6 / \pi^{2}\right) \alpha_{1}^{2}=\sin ^{2}\left(\delta-\delta_{W}\right)$. These two results substituted on its right-hand side, Eq. (48) reads

$$
G\left(\frac{T}{T_{K}}\right)-\frac{\mathcal{G}_{2}}{2}=-\left(G^{S}\left(\frac{T}{T_{K}}\right)-\frac{\mathcal{G}_{2}}{2}\right) \cos 2\left(\delta-\delta_{W}\right) .
$$

Central in this paper, Eq. (49) maps the conductance $G\left(T / T_{K}\right)$ to the universal function $G^{S}\left(T / T_{K}\right)$ linearly. The mapping is controlled by the argument $2\left(\delta-\delta_{W}\right)$ of the trigonometric function on the right-hand side. According to the Friedel sum rule, ${ }^{42} 2\left(\delta-\delta_{W}\right) / \pi$ is the screening charge induced by the coupling to the dot, which must equal the dot occupation $n_{d}$ to insure electrically neutrality. In particular, when the gate potential $V_{g}$ is such that dot occupation is unitary, the phase-shift difference is $\delta-\delta_{W}=\pi / 2$, the cosine on the right-hand side of Eq. (49) is equal to -1 , the second terms on the right- and left-hand sides cancel out of Eq. (49), and the remaining terms are equal, $G\left(T / T_{K}\right)=G^{S}\left(T / T_{K}\right)$. A particular case is the symmetric Hamiltonian (11), for which $\delta=\pi / 2, \delta_{w}=0$, and $G\left(T / T_{K}\right)=G^{S}\left(T / T_{K}\right){ }^{29}$
For other gate potentials in the Kondo regime, the groundstate dot occupation is approximately unitary, as Fig. 3 explained. The phase shift difference is never too far from $\pi / 2$. If, by contrast, it were $\delta-\delta_{w}=\pi / 4$, the conductance in Eq. (49) would be flat: $G(T)=\mathcal{G}_{2} / 2$. For the intermediate differences $\pi / 4<\delta-\delta_{w} \leqslant \pi / 2$ observed in the Kondo regime, the conductance lies between the universal curve $G^{S}\left(T / T_{K}\right)$ and the horizontal $\mathcal{G}_{2} / 2$. Although monotonically decreasing, the function $G\left(T / T_{K}\right)$ is therefore flatter than $G^{S}$.

Since $\delta-\delta_{w}$ is never too far from $\pi / 2$ in the Kondo regime, at the qualitative level we could still treat $G\left(T / T_{K}\right)$ as if it were proportional to $G^{S}\left(T / T_{K}\right)$, but the mapping (49) yields much more accurate conductances and affords quantitative comparison with numerical or experimental data. To underline this conclusion, the following sections present an NRG survey of electrical conduction through the device in Fig. 1.

\section{NUMERICAL PROCEDURE}

Equation (12) offers an approximation for $T_{K}$, and Eqs. (16), (19), and (21) roughly determine the ground-state phase shift $\delta$. Such estimates are far from the accuracy needed to fit numerical or experimental data. In the laboratory, $T_{K}$ and $\delta$ $-\delta_{W}$ are adjustable parameters. The former, in particular, is determined by the definition $G\left(T_{K}\right) \equiv \mathcal{G}_{2} / 2.5,32,33$

In the computer office, the two unknown parameters on the right-hand side of Eq. (49) can be extracted from the conductance itself, or from other properties of the model Hamiltonian. The phase shift $\delta$ can be obtained from the ground-state eigenvalues of $H_{A}$, or from the ground-state dot occupation. The Kondo temperature $T_{K}$ has been traditionally derived from fits of the temperature dependent magnetic susceptibility $\chi(T)$ with the universal curve for $k_{B} T \chi\left(T / T_{K}\right){ }^{2,4}$ Here, we break with the tradition and adopt the convention $G\left(T_{K}\right) \equiv \mathcal{G}_{2} / 2$ so that both sides of Eq. (49) vanish at $T=T_{K}$.

Once $T_{K}$ and $\delta$ have been determined, by either a Bethe ansatz calculation or an NRG computation, ${ }^{3,4,28}$ one can rely on the mapping (49) to evaluate the temperature-dependent conductance $G(T)$ in the Kondo regime. Alternatively, one can apply the NRG procedure described in Secs. VII A and VII B to compute equally accurate conductance curves $G(T)$ over the entire parametrical space of the model.

Here, we rely on the latter approach to provide a more comprehensive view of the model. Sections VIII A and VIII B will present NRG computations of the conductance as a function of the gate voltage and temperature. We cover the Kondo regime and the neighboring regions of the parametric space of the model to describe charge transport in the singleelectron transistor and to examine the behavior of the mapping (49) beyond the limits of the Kondo regime.

\section{A. Numerical-renormalization group method}

Excellent descriptions of the NRG method being available, ${ }^{2,28,46}$ one page will be sufficient to recapitulate the four constituents of the procedure.

\section{Logarithmic discretization}

Two dimensionless parameters $\Lambda>1$ and $0<z \leqslant 1$ define the logarithmic discretization of the conduction band. ${ }^{28,47}$ 
The infinite energy sequence $\mathbb{E}_{m}=D \Lambda^{1-z-m}(m=0,1, \ldots)$ defines the intervals $\mathbb{I}_{m}=\left[\mathbb{E}_{m+1}, \mathbb{E}_{m}\right]$. For each interval, a single operator $a_{m+}=\int_{\mathbb{I}_{m}} a_{k} d \varepsilon_{k} / n_{m}$, with normalization factor $n_{m}$, is defined. In the negative half of the conduction band, the sequence $-\mathbb{E}_{m}(m=0,1, \ldots)$ defines the mirror image $a_{m-}$ of each operator $a_{m+}$. The $a_{m \pm}$ form a basis upon which the conduction band Hamiltonian is projected. ${ }^{2}$

\section{Lanczos transformation}

Next, a Lanczos transformation ${ }^{48}$ makes tridiagonal the projected conduction Hamiltonian so that the model Hamiltonian reads

$$
H_{A}=\left(\sum_{n=0}^{\infty} t_{n} f_{n}^{\dagger} f_{n+1}+V f_{0}^{\dagger} c_{d}+\text { H.c. }\right)+W f_{0}^{\dagger} f_{0}+H_{d} .
$$

Here, $f_{0}$ is the operator defined in Eq. (6), and $f_{n}$ 's $(n$ $=0,1, \ldots)$ form an orthonormal basis that replaces $a_{m \pm}$ 's $(m=0,1, \ldots)$. With $z=1$, we recover the Lanczos transformation in Ref. 2. Otherwise, the codiagonal coefficients $t_{n}$ have to be determined numerically. ${ }^{47}$ With error $\mathcal{O}\left(\Lambda^{-n}\right)$, it is found $^{49}$ that $t_{n}=D\left[\left(1-\Lambda^{-1}\right) / \log \Lambda\right] \Lambda^{1-z-n / 2}$, a result that brings us to the third step in the NRG procedure, the definition of a truncated Hamiltonian.

\section{Infrared truncation}

Given a temperature $T$ and a small dimensionless parameter $\alpha$, let $N$ be the smallest integer such that $t_{N}<\alpha k_{B} T$ and consider the infinite sum on the right-hand side of Eq. (50). Compared to $k_{B} T$, the codiagonal element $t_{N}$ is then negligible. To compute $G(T)$, it is hence safe to drop the term with $n=N$ in the infinite series, an approximation that decouples the subsequent terms from the dot Hamiltonian. Once decoupled, the terms with $n>N$ will no longer contribute to the conductance; to neglect $t_{N}$ is thus equivalent to introducing an infrared cutoff $t_{N-1}$. Since $k_{B} T$ and $t_{N-1}$ are of the same order of magnitude, to emphasize that $k_{B} T$ sets the energy scale we define the reduced bandwidth $D_{N} \equiv D[(1$ $\left.\left.-\Lambda^{-1}\right) / \log \Lambda\right] \Lambda^{-(N-1) / 2}$ and the dimensionless, scaled, truncated Hamiltonian $H_{A}^{N}$,

$$
D_{N} H_{A}^{N} \equiv\left(\sum_{n=0}^{N-1} t_{n} f_{n}^{\dagger} f_{n+1}+V f_{0}^{\dagger} c_{d}+\text { H.c. }\right)+W f_{0}^{\dagger} f_{0}+H_{d} \text {. }
$$

In the scaled sum on the right-hand side, $t_{N-1} / D_{N}$, the smallest codiagonal coefficient, is of $\mathcal{O}(1)$.

\section{Iterative diagonalization and ultraviolet truncation}

The last step in the NRG procedure is the iterative diagonalization of the model Hamiltonian. With $N=0$, the righthand side of Eq. (51) is easily diagonalized; four eigenvalues $E_{m}^{0}$ and four eigenvectors $|m\rangle_{0}(m=1, \ldots, 4)$ result. At this stage, it is equally simple to calculate the matrix elements ${ }_{0}\left\langle m\left|c_{d}\right| n\right\rangle_{0}$ between the eigenvectors of $H_{A}^{N=0}$, which will be needed to compute the conductance.

Application of the operators $f_{0 \uparrow}^{\dagger}, f_{0 \downarrow}^{\dagger}, f_{0 \uparrow}^{\dagger} f_{0 \downarrow}^{\dagger}$, and the identity 1 on the eigenvectors of $H_{A}^{N=0}$ generates 16 states that constitute a basis upon which the Hamiltonian $H_{A}^{N=1}$ can be projected. Appropriately chosen linear combinations of those operators yield basis states $|p\rangle_{1}(p=1, \ldots, 16)$ that diagonalize the charge and spin operators; projected on them, $H_{A}^{N=1}$ reduces to block-diagonal matrices, which are then diagonalized numerically. The matrix elements ${ }_{0}\left\langle m\left|c_{d}\right| n\right\rangle_{0}(m, n$ $=1, \ldots, 4)$ are projected onto the basis $|p\rangle_{1}$ and subsequently rotated to the basis of the eigenstates $|m\rangle_{1}(m=1, \ldots, 16)$ of $H_{A}^{N=1}$. Application of the operators $f_{1 \uparrow}^{\dagger}, f_{1 \downarrow}^{\dagger}, f_{1 \uparrow}^{\dagger} f_{1 \downarrow}^{\dagger}$, and 1 on the $|m\rangle_{1}$ creates 64 basis vectors upon which $H_{A}^{N=2}$ can be projected, and the procedure is iterated.

To check the exponential growth of matrix dimensions, a dimensionless parameter $v$ is chosen, an ultraviolet cutoff that controls the cost and the accuracy of the iterative diagonalization. At the end of iteration $N$, the eigenvectors with scaled energies $E_{m} / D_{N}$ above $v$ are discarded before the construction of the basis states $|p\rangle_{N+1}$, upon which the Hamiltonian $H_{A}^{N+1}$ will be projected. This expedient limits the number of basis states and hence the computational effort in each iteration. The cost of a full NRG run grows linearly with the number of iterations.

\section{B. Computation of the conductance}

In each iteration, the diagonalization in Sec. VII A 4 yields the eigenvalues $E_{m}(m=0,1, \ldots, M$, where $M$ is an integer determined by the ultraviolet cutoff) and corresponding eigenvectors $|m\rangle$ of the scaled Hamiltonian (51). Once the matrix elements $\left\langle m\left|c_{d}\right| n\right\rangle(m, n=0,1, \ldots, M)$ are computed, one could in principle combine Eqs. (8) and (10) to compute the conductance at any temperature. In practice, in each iteration the infrared and ultraviolet truncations define a narrow window, in which the computed conductance can be accurately computed. This section explains how one can combine the sequence of thermal intervals thus resulting from the iterative diagonalization to compose a conductance curve.

\section{Relation between the conductance and the eigenvalues and eigenvectors of the scaled Hamiltonian}

Equation (8) relates the conductance to the dot-level spectral density $\rho_{d}(\epsilon, T)$. Since the temperature $T$ defines the energy window in each iteration, the thermal energy $k_{B} T$ is guaranteed to be between the infrared and ultraviolet cutoffs. Although $\epsilon$ may be above (below) the ultraviolet (infrared) cutoff, it is easy to see that only the energies inside the window contribute significantly to the conductance. To this end, substitute Eq. (10) on the right-hand side of Eq. (8). The resulting integral leads to an expression for the conductance that depends only on the eigenvalues $E_{m}$ of $H_{A}^{N}$ within the energy window and on the corresponding eigenvectors $|m\rangle$,

$$
G(T)=\mathcal{G}_{2} \frac{\beta \pi \Gamma_{w}}{\mathcal{Z}} \sum_{m n} \frac{\left|\left\langle m\left|c_{d}\right| n\right\rangle\right|^{2}}{e^{\beta E_{m}}+e^{\beta E_{n}}} .
$$

As suggested by this equality, which can be reduced to a few lines of computer code, the computational effort behind a conductance curve $G(T)$ is comparable to the cost of a magnetic susceptibility plot. $^{2}$ 


\section{Thermal ranges}

The eigenvalues of the scaled truncated Hamiltonian range from unity to $v$ and hence correspond to energies ranging from $D_{N}$ to $v D_{N}$. Having neglected $t_{N} \approx D_{N+1}$, we can only compute conductances for $k_{B} T>\gamma D_{N+1}$, where $\gamma \gtrsim 10$. At the other extreme, the ultraviolet truncation restricts us to temperatures such that $k_{B} T<v D_{N}$. Thus, provided that $\left(D_{N} v\right) /\left(\gamma D_{N+1}\right)>\sqrt{\Lambda}$, i.e., that $v>\gamma$, the $N$ th iteration yields reliable conductances in the temperature window $\gamma D_{N+1}$ $\leqslant k_{B} T \leqslant \sqrt{\Lambda} \gamma D_{N+1}$. If a run is stopped at iteration $N_{\max }$, the juxtaposition of the resulting windows yields $G(T)$ for all temperatures above $\gamma D_{N_{\max }+1} / k_{B}$. In practice, the conductance is only computed for the $k_{B} T \leqslant 0.1 D$ because irrelevant operators artificially introduced by the logarithmic discretization make the interval $0.1 D<k_{B} T \leqslant D$ unreliable.

\section{3. $z$ trick}

Conductance curves computed with large $\Lambda$ show oscillations, which can be traced to a sequence of poles on the $\mathfrak{J} \epsilon$ $= \pm i \pi / \log \Lambda$ lines of the complex-energy plane. ${ }^{47}$ To eliminate these artifacts of the discretization, we average the conductance curve $G(T)$ computed for given $z$ over a sequence of equally spaced $z$ 's in the interval $0<z \leqslant 1 .^{50}$ The exponential dependence of the computational effort on $1 / \log \Lambda$ makes this averaging procedure far more efficient than comparably accurate computations with small $\Lambda$.

\section{Numerics}

The conductances in Sec. VIII were computed with $\Lambda$ $=6$ and averaged over two z's: 0.5 and 1. The amplitude of the residual oscillations encountered after averaging over $z$, somewhat smaller than $0.001 e^{2} / h$, provides an estimate of the error introduced by the logarithmic discretization. The other two parameters controlling the precision of the results were fixed at $\gamma=10.5$ and $v=50$, respectively. Spin degeneracies not counted the number of states below the cutoff in each iteration peaked at 4000 in iteration 6 . To estimate the error due to the infrared and ultraviolet truncations, for each $N<N_{\max }$ we compared the conductance at the lowest temperature in the $(N-1)$ th window, $\gamma D_{N} \leqslant k_{B} T \leqslant \sqrt{\Lambda} \gamma D_{N}$, with the conductance at the highest temperature in the $N$ th window. The mismatch between the two results never exceeding $0.001 e^{2} / h$, we conclude that deviations due to the three approximations in the procedure, the logarithmic discretization and the infrared and ultraviolet truncations, are comparable. At any temperature, the estimated absolute deviation in the computed conductances is smaller than $0.05 \%$ of the quantum conductance.

The relatively large discretization parameter expedites the calculation. On a standard desktop computer, a complete run, including (i) the iterative diagonalization of $H_{A}$ and computation of the matrix elements on the right-hand side of Eq. (52) for each $z$ and (ii) the evaluation of the conductance curve in the interval $10^{-10} D<k_{B} T \leqslant 0.1 D$, takes less than $30 \mathrm{~s}$.

\section{Renormalization-group flow}

Along with the iterative diagonalization procedure, Eq. (51) defines a renormalization-group transformation, ${ }^{2}$

$$
\mathbb{T}\left[H_{A}^{N}\right] \equiv H_{A}^{N+2}=\Lambda H_{A}^{N}+\sum_{n=N}^{N+1} \frac{t_{n}}{D_{N+2}}\left(f_{n}^{\dagger} f_{n+1}+\text { H.c. }\right) .
$$

The factor $\Lambda$ multiplying the first term on the right-hand side magnifies the scale on which the eigenvalues of $H_{A}^{N}$ are examined. On the new scale, the second term is a fine structure. In the absence of characteristic energies, as $N$ grows the magnification compensates the refinement, and the lowestenergy eigenvalues of $H_{A}^{N+2}$ rapidly become indistinguishable from those of $H_{A}^{N}$. This indicates that the Hamiltonian has reached a fixed point of $T$.

In the Kondo regime, the condition $V=0$ turns the Anderson Hamiltonian $H_{A}$ into the LM fixed point of T. With $V$ $\neq 0$, as the temperature is reduced past the dominant characteristic energy $E_{c}=\min \left(\left|\varepsilon_{d}\right|, \varepsilon_{d}+U, D\right)$, the Hamiltonian $H_{A}^{N}$ first approaches the LM and then moves away toward the FL fixed point-a strong-coupling fixed point equivalent to Eq. (5) with $V \rightarrow \infty$. Between the LM and the FL lies the Kondo temperature $T_{K}$, around which the conduction electrons screen the dot moment.

If one of the dot-charge excitation energies, $\Delta_{0} \equiv\left|\varepsilon_{d}\right|$ or $\Delta_{2} \equiv \varepsilon_{d}+U$, is smaller than the dot width $\Gamma_{w}$, the model Hamiltonian enters the mixed-valence regime. ${ }^{55}$ Instead of $\min \left(D,\left|\varepsilon_{d}\right|, \mid e d+U\right)$, the dominant characteristic energy is now $E_{c}=\min \left(D, \Gamma_{w}\right)$. The dot moment is only partially formed, as the coupling $\Gamma_{w}$ drives the model Hamiltonian toward the FL before it can come close to the LM.

\section{Phase shifts}

The potential $W_{L M}\left(W_{F L}\right)$ on the right-hand side of Eq. (14) [Eq. (15)] and the associated phase shift $\delta_{L M}(\delta)$ depend on the model parameters. With $\Gamma=0$, for instance, the Hamiltonian $H_{A}$ flows directly toward the LM; therefore, $W_{L M}$ $=W$ and $\delta_{L M}=\delta_{w}$. For $0<\Gamma \ll D$, approximate LM phase shifts can be extracted from the eigenvalues of $H_{A}^{N}$, where $N$ is such that $D \gg D_{N} \gg k_{B} T_{K}$, i.e., such that the model Hamiltonian dwells in the vicinity of the LM. For all $\Gamma>0$, by contrast, the FL phase shift $\delta$ can be calculated very accurately from the low-energy eigenvalues of $H_{A}$ because as $N$ $\rightarrow \infty$ the truncated Hamiltonian approaches the FL Hamiltonian

$$
H_{F L}^{*}=\sum_{\ell, \pm} \varepsilon_{\ell \pm}^{*} g_{\ell \pm}^{\dagger} g_{\ell \pm} \cdot
$$

Here, + and - subscripts distinguish the positive eigenvalues from the negative ones, while $\ell=0,1, \ldots$ counts the positive (negative) eigenvalues upward (downward) from the Fermi level.

Once the eigenvalues of $H_{A}^{N}$ are identified with the manybody energies generated from Eq. (15), the ground-state phase shift $\delta$ are extracted from the following approximate expression, which describes all but the $\varepsilon_{\ell_{ \pm}}^{*}$ closest to zero very accurately. ${ }^{45}$ For $\Lambda \geqslant 5$, in particular, within $0.1 \%$ deviation,

$$
\varepsilon_{\ell \pm}^{*}= \pm \Lambda^{\nu+\ell \mp \delta / \pi} \quad(\ell=1,2, \ldots),
$$

where $\nu=1-z(\nu=3 / 2-z)$ for odd (even) $N$. 


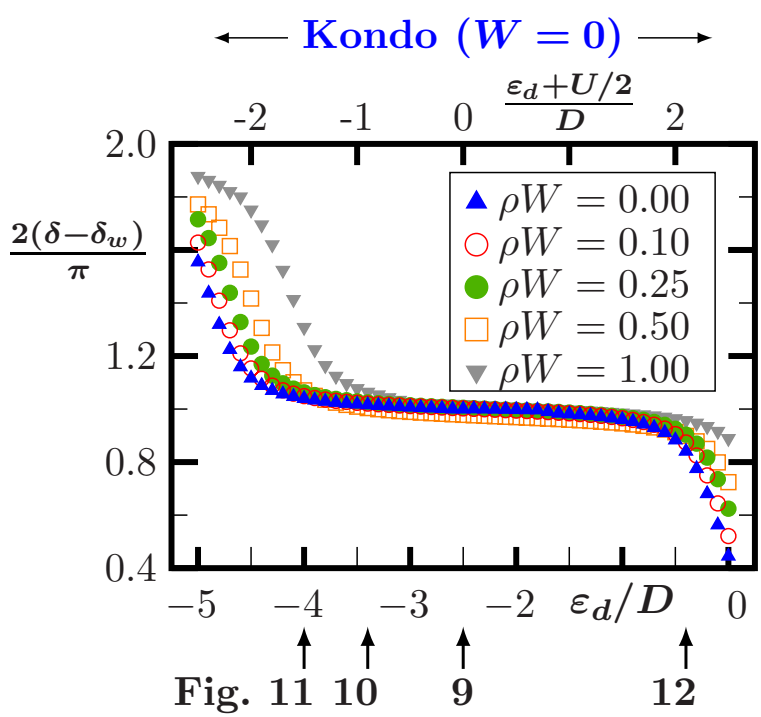

FIG. 6. (Color online) Ground-state phase shift $\delta$, measured from the phase shift $\delta_{w}$ obtained from Eq. (B6) for the displayed wire potentials $W$, as a function of the dot-level energy $\varepsilon_{d}$. The phase shifts are defined in the domain $0<\delta \leqslant \pi$ so that the Friedel sum rule makes the ordinate equal the dot occupation $n_{d}$. The $\delta$ s were obtained, with the help of Eq. (55), from the low-energy spectrum of $H_{A}$ resulting from NRG runs with $U=5 D$ and $\Gamma_{w}=0.15 D$. The arrows above the top horizontal axis define the Kondo domain for $W=0$. For $W \neq 0$, the Kondo domain is displaced to the right. Each vertical arrow pointing to the lower horizontal axis identifies the figure displaying the thermal dependence of the conductance for the indicated dot energy.

\section{RESULTS}

To emulate the conditions under which a SET operates, we fix the Coulomb repulsion $U$ and effective dot-level width $\Gamma_{w}$ and examine the ground-state phase shift and the thermal dependence of the conductance as a function of the dot energy $\varepsilon_{d}$ for fixed wire potential $W$. To mimic the parameters describing typical devices, Sec. VIII B will study model Hamiltonians with $U<D$. The opposite inequality is, however, more illustrative because it displaces the Kondo regime to higher temperatures. We prefer to discuss it first.

\section{A. Conductance for $U>D$}

To display a broad range of Kondo temperatures, we choose the Coulomb repulsion $U=5 D$ and the effective dotlevel width $\Gamma_{w}=0.15 D$ and examine the ground-state phase shift $\delta$ and thermal dependence of the conductance $G(T)$ as functions of the dot energy $\varepsilon_{d}$ for five positive wire gate potentials $W$. We need not study negative wire potentials, which would mirror the conductances and phase shifts calculated with positive $W$, because $H_{A}, G$, and $|\delta|$ remain invariant under the transformation $c_{d} \rightarrow-c_{d}^{\dagger}, a_{k} \rightarrow a_{k}^{\dagger}, \varepsilon_{d}+U / 2$ $\rightarrow-\left(\varepsilon_{d}+U / 2\right)$, and $W \rightarrow-W$.

Consider, first, the phase shift. Figure 6 displays the argument of the trigonometric function on the right-hand side of the mapping (49), computed for five wire potentials $W$, in the dot-energy range $0 \geqslant \varepsilon_{d} / D \geqslant-U$. To draw continuous curves and to make the ordinate $2\left(\delta-\delta_{w}\right) / \pi$ equal to the ground- state dot occupancy $n_{d}$, we have displaced the domain of definition of $\delta$ from $(-\pi / 2, \pi / 2)$ to $(0, \pi)$,

Calculated for $W=0$, the upright triangles trace a wellknown curve, ${ }^{4}$ one that remains invariant under the particlehole transformation $\varepsilon_{d}+U / 2 \rightarrow-\left(\varepsilon_{d}+U / 2\right), \delta \rightarrow \pi / 2-\delta$. At the symmetric point $\varepsilon_{d}+U / 2=0$, which corresponds to Eq. (11), the phase shift is exactly $\pi / 2$. The arrows above the top axis indicate the Kondo domain, within which the phase shift remains close to $\pi / 2$. As $\left|\varepsilon_{d}+U / 2\right|$ grows, the model Hamiltonian first approaches the limits of the Kondo domain and then invades the mixed-valence domain. In response, $\delta$ moves away from $\pi / 2$, toward zero for $\varepsilon_{d}+U / 2 \rightarrow U / 2$, or toward $\pi$ for $\varepsilon_{d}+U / 2 \rightarrow-U / 2$.

The wire potential reduces the ground-state phase shift throughout the depicted range. For $\rho W=1$, for instance, the phase shift at the symmetric dot-level energy $\varepsilon_{d}=-2.5 D=$ $-U / 2$ is reduced from $\delta=\pi / 2$ to $\delta=\pi / 10$. In the Kondo regime, as the illustration shows, the difference $\delta-\delta_{w}$ is nonetheless pinned at $\pi / 2$. The pinning is due to the Friedel sum rule. ${ }^{42}$ Since the ground-state phase shift would be $\delta_{w}$ if $\Gamma$ were zero, $2\left(\delta-\delta_{w}\right) / \pi$ is the screening charge due to the coupling to the dot. In the Kondo regime, that charge is nearly unitary, and $\delta-\delta_{w} \approx \pi / 2$.

Figure 6 also shows that a positive wire potential tends to displace the Kondo regime toward higher dot energies. For $W=0$, the rapid decay of the phase shift near $\varepsilon_{d}=0 \quad\left(\varepsilon_{d}=\right.$ $-U)$ marks the resonance between the $n_{d}=0$ and $n_{d}=1\left(n_{d}\right.$ $=1$ and $n_{d}=2$ ) dot-level configurations. The Kondo regime lies between them. As $W$ grows, the two resonances move to higher $\varepsilon_{d}$ 's and so does the Kondo regime.

\section{Conductance landscape}

According to Eq. (49), the phase-shift difference $\delta-\delta_{w}$ controls $G(T)$. Consequently, the central features of Fig. 6 are manifest in landscape plots of the conductance. Figure 7 (a) shows $G(T)$ in the dot-energy range $\left|\varepsilon_{d}+U / 2\right| \leqslant U / 2$ for $U=5 D$, and $\Gamma=0.15 D$. The plot surveys the entire Kondo regime and part of the mixed-valence regime. The plane $\varepsilon_{d}$ $=-U / 2$, which represents the symmetric Hamiltonian (11), splits the landscape in two symmetric halves, mapped onto each other by the particle-hole transformation $c_{d} \rightarrow-c_{d}^{\dagger}, a_{k}$ $\rightarrow a_{k}^{\dagger}$.

At the symmetric point $\varepsilon_{d}=-U / 2$, the temperaturedependent conductance reproduces the universal function $G^{S}\left(T / T_{K}\right)$. Here and elsewhere in the Kondo regime, the conductance at fixed $\varepsilon_{d}$ rises from zero to ballistic as the temperature is reduced past $T_{K}$, i.e., as one climbs from the hightemperature Coulomb-blockade valley to the lowtemperature Kondo plateau.

The Kondo temperature depends on $\varepsilon_{d}$. Plotted in Fig. 7 (b) as a function of the dot energy, $T_{K}$ mirrors the invariance of the Hamiltonian under particle-hole transformations and reaches the minimum $k_{B} T_{K}=8 \times 10^{-7} \mathrm{D}$ at the symmetric point. As $\left|\varepsilon_{d}+U / 2\right|$ grows, the Kondo temperature rises until $k_{B} T_{k} \approx 0.1 D$, an equality indicating proximity to the mixedvalence regime, i.e., to the two resonances centered at $\varepsilon_{d}=$ $-5 D$ and $\varepsilon_{d}=0$. As $\left|\varepsilon_{d}+U / 2\right|$ grows further, we come into mixed-valence domain. The dot moment shrinks, and so does the Kondo cloud. The Kondo bypass of the Coulomb block- 


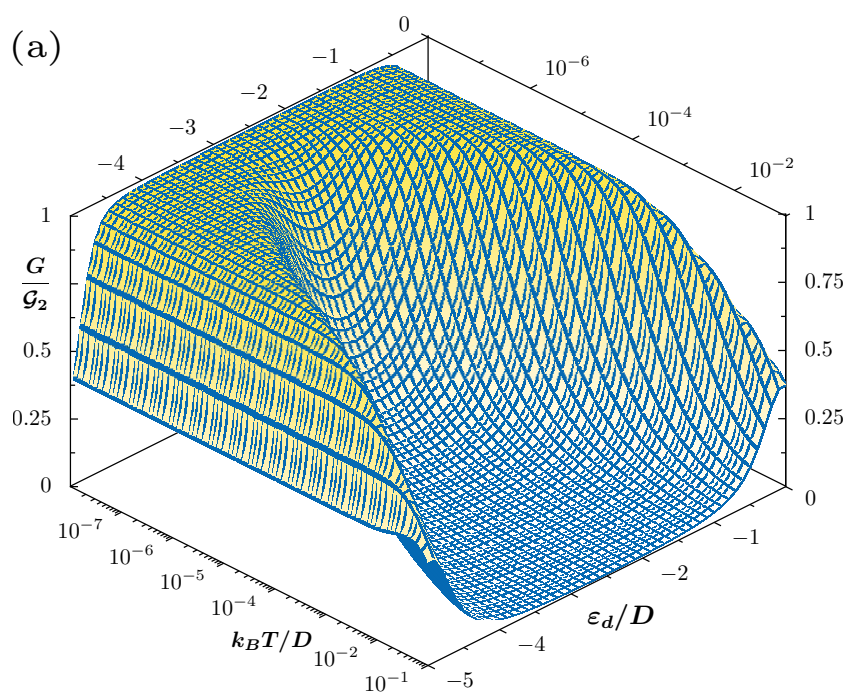

(b)

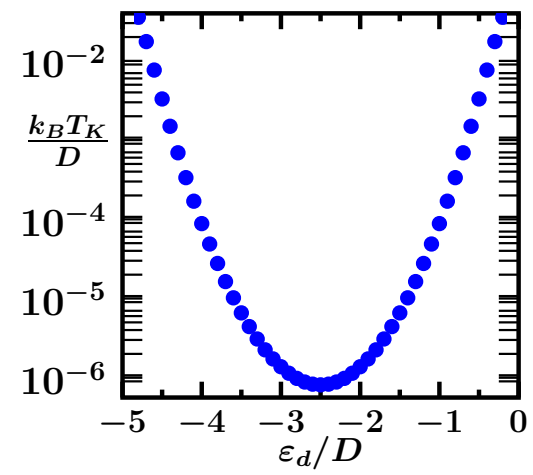

FIG. 7. (Color online) (a) Conductance as a function of the temperature and dot-level energy for $U=5 D, \Gamma=0.15 D$, and $W=0$. The plot is symmetric with respect to the $\varepsilon_{d}=-U / 2=-2.5 D$ plane. The sharp drops near $\varepsilon_{d}=-5 D$ and $\varepsilon_{d}=0$ mark the borders of the Kondo regime, which extends roughly from $\varepsilon_{d}=-\Gamma$ to $\varepsilon_{d}+U=-\Gamma$. In the Kondo regime, at fixed $\varepsilon_{d}$, the more gradual decay of the conductance with temperature portrays the evaporation of the Kondo droplet. (b) Kondo temperatures resulting from the intersection of the landscape (a) with the horizontal plane $G\left(T=T_{K}\right)$ $\equiv \mathcal{G}_{2} / 2$.

ade becomes less and less effective, and the conductance approaches zero. The steep drops near the $\varepsilon_{d}=0$ and $\varepsilon_{d}=$ $-5 D$ planes in Fig. 7(a) mark the fractional dot occupations $n_{d} \approx 0.5$ and $n_{d} \approx 1.5$, respectively.

\section{Wire potential}

Figure 8(a) displays the conductance as a function of $\varepsilon_{d}$ and $T$ for $U=5 D, \Gamma_{w}=0.15 D$, and $\rho W=1$. Quantitative differences distinguish the plot from Fig. 7(a). In particular, the Kondo temperature is now minimized at the higher dot-level energy $\varepsilon_{d}=-1.9 D$, the minimum is 30 -fold higher, $k_{B} T_{K}$ $=2.4 \times 10^{-6} \mathrm{D}$, the resonance between the $n_{d}=1$ and $n_{d}=2 \mathrm{dot}$ configurations is now centered at $\varepsilon_{d} \approx-4.2 D$, and of the resonance between the $n_{d}=0$ and $n_{d}=1$ dot configurations only an incipient rise is visible at the high- $\varepsilon_{d}$ end of the plot. Clearly, the wire potential has displaced the Kondo domain toward higher dot-level energies. This displacement ac-

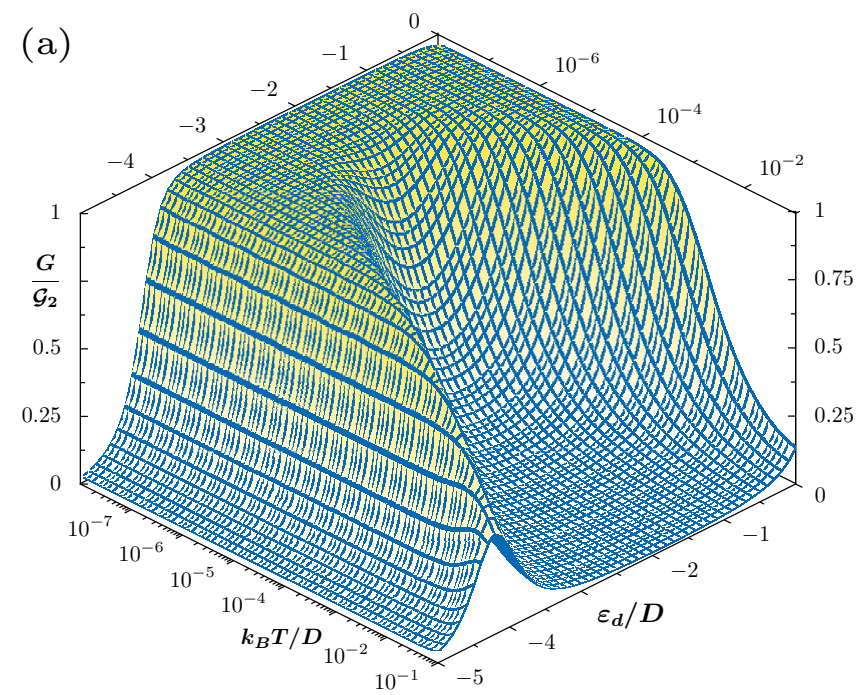

(b)

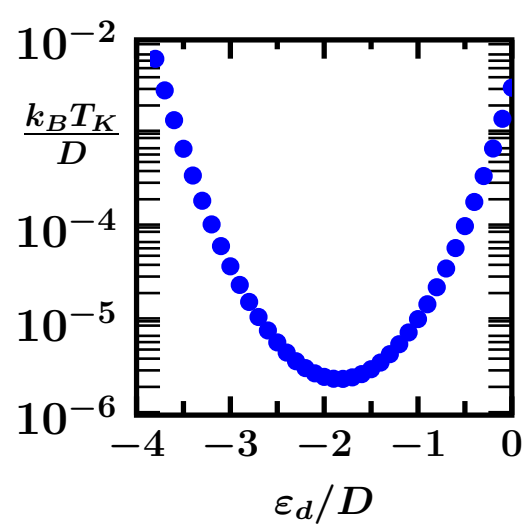

FIG. 8. (Color online) (a) Conductance as a function of the temperature and dot-level energy for $U=5 D, \Gamma_{w}=0.15 D$, and $\rho W$ $=0.50$. The wire potential breaks the particle-hole symmetry visible in Fig. 7. The sharp drop centered at $\varepsilon_{d}=-5 D$ in Fig. 7 is now fully visible, while the one centered at $\varepsilon_{d}=0$ is out of sight, an indication that the Kondo regime has been displaced to higher dot energies. The bell-shaped resonance near the bottom left corner of the $k_{B} T$ $=10^{-1} \mathrm{D}$ plane stakes the mixed-valence regime. (b) Kondo temperatures resulting from the intersection of the landscape (a) with the plane $G\left(T=T_{K}\right)=\mathcal{G}_{2} / 2$.

knowledged, we recognize in Fig. 8(a) the salient features of Fig. 7(a).

The two landscapes are similar because the dependence relating the phase-shift difference on the right-hand side of Eq. (49) to the wire potential $\rho W$ is weak. With $\delta-\delta_{w}$ $\approx \pi / 2$, the conductance curve $G\left(T / T_{K}\right)$ is approximately mapped onto $G^{S}\left(T / T_{K}\right)$ throughout the Kondo domain. The rise from the high-temperature valley to the Kondo plateau is therefore close to universal, dependent on the model parameters only through the Kondo temperature $T_{K}$.

Figure 8(b) shows the Kondo temperature as a function of the dot energy, a plot that resembles Fig. 7(b). $T_{K}$ depends on the antiferromagnetic interaction $J$ between the dot moment and the conduction electrons around it, a constant related to the model parameters by the Schrieffer-Wolff expression, ${ }^{41}$ 


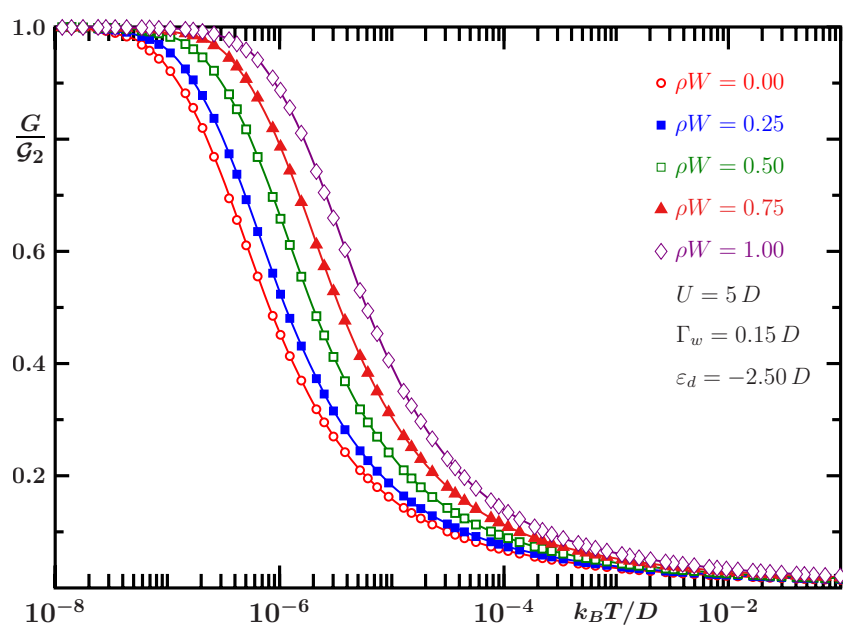

FIG. 9. (Color online) Thermal dependence of the conductance for $\varepsilon_{d}+U / 2=0$, and the indicated values of the other model parameters. The circles, open and filled squares, triangles, and diamonds are the NRG data, while the solid lines through them depict Eq. (49), with the Kondo temperatures and phase shifts listed in Table I. The curve through the open circles, in particular, is the universal conductance $G^{S}\left(T / T_{K}\right)$ for the symmetric Hamiltonian (11) (Ref. $29)$. Since $\left|\delta-\delta_{w}\right| \approx \pi / 2$, each solid line is close to $G^{S}\left(T / T_{K}\right)$.

$$
\rho J=\frac{2 \Gamma_{w}}{\pi}\left(\frac{1}{\Delta_{0}}+\frac{1}{\Delta_{2}}\right) .
$$

Here, $\Delta_{0}\left(\Delta_{2}\right)$ is one of the two dot-charge excitation energies, the energy needed to remove (add) an electron to the singly-occupied dot level. For the symmetric Hamiltonian (11), in particular, $\Delta_{0}=\Delta_{2}=U / 2$. For nearly symmetric Hamiltonians, $\Delta_{0}=\left|\varepsilon_{d}\right|$ and $\Delta_{2}=U+\varepsilon_{d}$. As $|W|$ or $\left|\varepsilon_{d}+U / 2\right|$ grow, the resulting particle-hole asymmetry renormalizes the dot energy ${ }^{51,45}$ so that $\Delta_{0}$ and $\Delta_{2}$ are changed to $\Delta_{0}^{*}=\left|\varepsilon_{d}^{*}\right|$ and $\Delta_{2}^{*}=U+\varepsilon_{d}^{*}$, respectively, where $\varepsilon_{d}^{*}$ is the effective dot energy at the LM. ${ }^{45}$

Since both landscapes were computed for the same effective width $\Gamma_{w}=0.15 D$, only (i) the excitation energies $\Delta_{0}^{*}$ and $\Delta_{2}^{*}$ and (ii) irrelevant operators make the Kondo temperatures in Fig. 8(b) different from those in Fig. 7(b). The renormalized excitation energies displace the Kondo domain along the $\varepsilon_{d}$ axis, while the modified irrelevant operators extend the Kondo plateau toward higher temperatures.

This concludes our overview of the numerically computed conductance landscapes. Section VIII A 3 will inspect in more detail the data in four slices of Figs. 7(a) and 8(a) and compare them to Eq. (49).

\section{Thermal dependence of the conductance}

Figure 9 displays the conductance as a function of the temperature for $U=5 D, \Gamma_{w}=0.15 D, \varepsilon_{d}+U / 2=0$, and five wire potentials: $\rho W=0$ and 1 , already studied in Figs. 7 and 8 , and three intermediate values, $\rho W=0.25,0.5$, and 0.75 . With $W=0$, the open circles represent the symmetric Hamiltonian (11), and the solid line through them, the universal function $G^{S}\left(T / T_{K}\right)$ first computed in Ref. 29. Notwithstanding the wire potentials, the Hamiltonians represented by the squares, triangles, and diamonds lie deep inside the Kondo regime. For each of them, the phase-shift difference $\left|\delta-\delta_{w}\right|$ in Table I is close to $\pi / 2$. It follows that the right-hand side of Eq. (49) is close to $G^{S}\left(T / T_{K}\right)$. The agreement with the numerical data is excellent.

As the Hamiltonian moves away from the symmetric plane $\varepsilon_{d}=-U / 2$, the particle-hole asymmetry becomes more pronounced, and one might expect the phase shift difference $\left|\delta-\delta_{w}\right|$ to grow. As Fig. 6 showed, however, the growth is checked by the Friedel sum rule so that $\left|\delta-\delta_{w}\right| \approx \pi / 2$ in the Kondo regime. Illustrative results appear in Fig. 10, which displays conductance curves for $\varepsilon_{d}=-3.4 D$. Even for the strongest wire potential in the legend, $\rho W=1$, the difference $\left|\delta-\delta_{w}\right|$ in Table I is only $6 \%$ away from $\pi / 2$. As in Fig. 9, therefore, the conductance curves computed from Eq. (49) are nearly identical to $G^{S}\left(T / T_{K}\right)$. The agreement with the numerical data is again excellent. Since we are now closer to the boundary of the Kondo regime, the Kondo temperature is more sensitive to the renormalization of the dot-level energy induced by strong wire potentials. Compared to Fig. 9, Fig. 10 thus exhibits a substantially broader spread of crossover temperatures.

Figure 11 displays numerical results for $\varepsilon_{d}+U / 2=-1.5 D$, a still larger departure from the symmetric condition. For $\rho W \leqslant 0.5$, the agreement with Eq. (49) is excellent; for $\rho W$ $=0.75$, it is imperfect only at the highest temperatures shown. For $\rho W=1$, however, there is substantial disagreement, which justifies a digression.

Inspection of Fig. 8 shows that for $\varepsilon_{d}=-4 D$ (and $\rho W=1$ ), the model Hamiltonian lies well within the mixed-valence regime. ${ }^{56}$ In the Kondo regime, Eq. (49) is reliable for thermal energies that are small on the scale of the dominant characteristic energy $E_{C}=\min \left(\left|\varepsilon_{d}^{*}\right|, U+\varepsilon_{d}^{*}, D\right)$. If $\varepsilon_{d}^{*}$ had its bare value, $\varepsilon_{d}=-4 D$, the mapping would be reliable for $k_{B} T \ll E_{c}=D$. The renormalized dot energy having pushed the model Hamiltonian into the mixed-valence regime, the dominant characteristic energy has been reduced to $E_{c}$ $=\min \left(\Gamma_{w}, D\right)=\Gamma_{w}$, which restricts the domain of the mapping to $k_{B} T \ll 0.15 D$. The failure at higher temperatures is due to the contribution $\Delta G_{\text {irr }}$ of irrelevant operators, which are sizable near the characteristic energy. At $k_{B} T=0.1 D=2 / 3 \Gamma_{w}$, for example, the diamonds in Fig. 11 are pushed $0.2 e^{2} / h$ below the solid line; upon cooling, $\Delta G_{\text {irr }}$ decays in proportion to $k_{B} T$ and becomes insignificant below $k_{B} T=10^{-2} D$.

If $-\varepsilon_{d}$ were steadily increased beyond $-\varepsilon_{d}=4 D$, the model Hamiltonian would traverse the mixed-valence region. Once $\left|\varepsilon_{d}^{*}+U\right|>\Gamma_{w}$, the dot occupation would approach $n_{d}=2$. The dominant characteristic energy $E_{c}=\left|\varepsilon_{d}^{*}+U\right|$ would define the crossover energy scale, which would hence rise with $-\varepsilon_{d}$. Soon, the model Hamiltonian would be driven to the frozenlevel fixed point at the first steps of the renormalizationgroup flow, and the mapping would be reduced to its FL limit, $G(T \rightarrow 0)=\sin ^{2}\left(\delta-\delta_{w}\right) \approx 0$.

Equation (49) is asymptotically exact at low temperatures, i.e., for $k_{B} T \ll E_{c}$. As the above discussion showed, its practical value is eroded outside the Kondo regime. In the mixedvalence regime, in particular, the asymptotic region lies well below the crossover temperature, i.e., in the vicinity of the FL. To plot the rightmost solid line in Fig. 11, we thus had to match the right-hand side of Eq. (49) to the diamond at $G$ 
TABLE I. Phase shifts and Kondo temperatures for the 20 NRG runs depicted in Figs. 9-12. The ground-state phase shifts $\delta$ were obtained from Eq. (55), the wire phase shifts $\delta_{w}$ from Eq. (B6), and the Kondo temperatures from the definition $G\left(T=T_{K}\right) \equiv \mathcal{G}_{2} / 2$. The Kondo temperature marked with an asterisk belongs to the mixed-valence regime and, as explained in the text, is the output of a different computation.

\begin{tabular}{|c|c|c|c|c|c|c|}
\hline Figure & Symbol & $\rho W$ & $\delta_{w} / \pi$ & $\delta / \pi$ & $\left|\delta-\delta_{w}\right| / \pi$ & $k_{B} T_{K} / D$ \\
\hline 9 & 0 & 0.00 & 0.00 & -0.50 & 0.50 & $8.1 \times 10^{-7}$ \\
\hline 9 & $\boldsymbol{\square}$ & 0.25 & -0.21 & 0.29 & 0.50 & $1.1 \times 10^{-6}$ \\
\hline 9 & $\square$ & 0.50 & -0.32 & 0.18 & 0.50 & $2 \times 10^{-6}$ \\
\hline 9 & $\Delta$ & 0.75 & -0.37 & 0.13 & 0.51 & $3.4 \times 10^{-6}$ \\
\hline 9 & $\diamond$ & 1.00 & -0.40 & 0.11 & 0.51 & $6 \times 10^{-6}$ \\
\hline 10 & $\bigcirc$ & 0.00 & 0.00 & -0.49 & 0.49 & $4.4 \times 10^{-6}$ \\
\hline 10 & $\square$ & 0.25 & -0.21 & 0.30 & 0.51 & $1.1 \times 10^{-5}$ \\
\hline 10 & $\square$ & 0.50 & -0.32 & 0.20 & 0.52 & $3.6 \times 10^{-5}$ \\
\hline 10 & $\Delta$ & 0.75 & -0.37 & 0.15 & 0.52 & $1.1 \times 10^{-4}$ \\
\hline 10 & $\diamond$ & 1.00 & -0.40 & 0.13 & 0.53 & $3.6 \times 10^{-4}$ \\
\hline 11 & 0 & 0.00 & 0.00 & -0.48 & 0.48 & $8.8 \times 10^{-5}$ \\
\hline 11 & $\mathbf{\square}$ & 0.25 & -0.21 & 0.32 & 0.53 & $3.3 \times 10^{-4}$ \\
\hline 11 & $\square$ & 0.50 & -0.32 & 0.23 & 0.55 & $1.6 \times 10^{-3}$ \\
\hline 11 & $\boldsymbol{\Delta}$ & 0.75 & -0.37 & 0.22 & 0.59 & $7.3 \times 10^{-3}$ \\
\hline 11 & $\diamond$ & 1.00 & -0.40 & 0.25 & 0.65 & $3.4 \times 10^{-2} *$ \\
\hline 12 & $\bigcirc$ & 0.00 & 0.00 & 0.42 & 0.42 & $8.0 \times 10^{-3}$ \\
\hline 12 & $\mathbf{\square}$ & 0.25 & -0.21 & 0.24 & 0.45 & $2.4 \times 10^{-3}$ \\
\hline 12 & $\square$ & 0.50 & -0.32 & 0.15 & 0.46 & $9.4 \times 10^{-4}$ \\
\hline 12 & $\Delta$ & 0.75 & -0.37 & 0.10 & 0.47 & $4.0 \times 10^{-4}$ \\
\hline 12 & $\diamond$ & 1.00 & -0.40 & 0.08 & 0.48 & $1.9 \times 10^{-4}$ \\
\hline
\end{tabular}

$=0.7 \mathcal{G}_{2}$ because the identification $G\left(T=T_{K}\right)=0.5 \mathcal{G}_{2}$, which defined $T_{K}$ for all the other plots in Figs. 9-12, became unreliable for $\rho W=1$. Table I marks with an asterisk the resulting Kondo temperature.

Near the opposite extreme of the Kondo regime, for fixed small $-\varepsilon_{d}$, the wire potential drives the model Hamiltonian

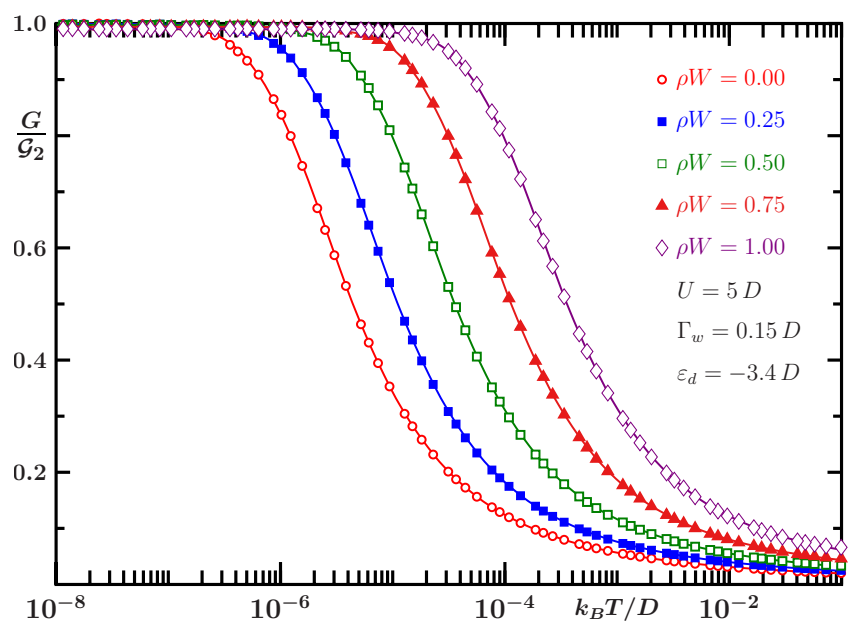

FIG. 10. (Color online) Thermal dependence of the conductance for $\varepsilon_{d}+U / 2=-0.9 D$. The symbols and lines were computed as described in Fig. 9. As Table I shows, the argument $\left|\delta-\delta_{w}\right|$ on the right-hand side of Eq. (49) is close to $\pi / 2$. As a consequence, the solid lines are only slightly different from $G^{S}\left(T / T_{K}\right)$. The agreement with the numerical data is, again, excellent. toward the center of the Kondo regime. Clear evidence of this displacement is found in Fig. 8(a): displaced to positive dot-level energies, the mixed-valence domain is no longer visible on the right-hand side of the landscape. The temperature dependence of the conductance is displayed in Fig. 12, which shows the $\varepsilon_{d}=-0.4 D$ plane for the five potentials $\rho W=0,0.25,0.5,0.75$, and 1 . The $\rho W=0$ Hamiltonian is

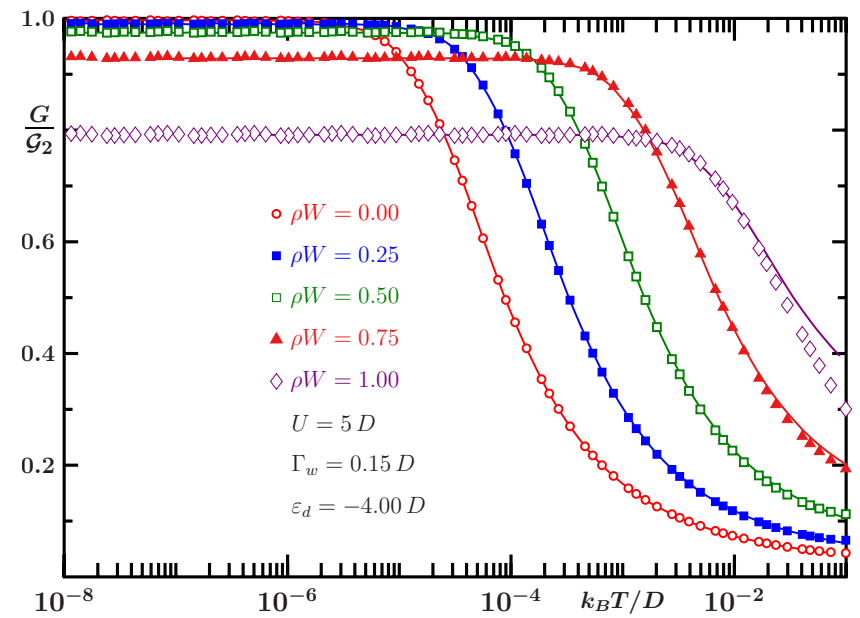

FIG. 11. (Color online) Thermal dependence of the conductance for $\varepsilon_{d}+U / 2=-1.5 D$. The symbols and lines were calculated as described in Fig. 9. As discussed in the text, the high-temperature separation between the solid line and the diamonds flags a Hamiltonian outside the Kondo regime. 


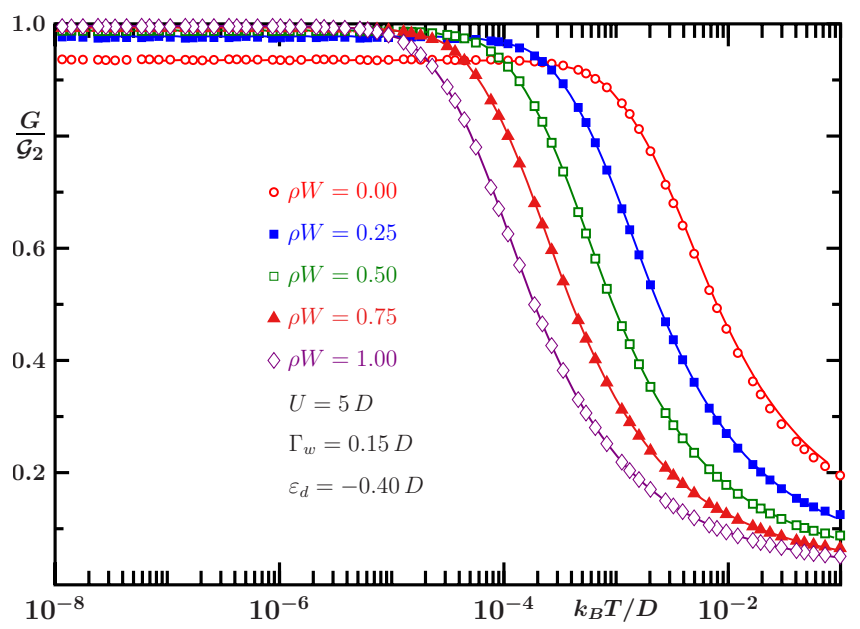

FIG. 12. (Color online) Thermal dependence of the conductance for $\varepsilon_{d}+U / 2=2.1 D$. The lines and symbols were computed as described in Fig. 9. The relatively large separation from the symmetric condition $\varepsilon_{d}+U / 2=0$ places the $W=0$ Hamiltonian close to the border of the Kondo regime; at high temperatures, relatively large irrelevant operators, whose influence decays in proportion to $k_{B} T / D$, introduce deviations from Eq. (49). Since the wire potential displaces the Kondo regime to higher dot-level energies, the distance from the border grows with $\rho W$, and so does the agreement between the numerical data and the solid lines representing Eq. (49).

now at the boundary of the Kondo regime, and for $k_{B} T$ $>10^{-2} \mathrm{D}$, irrelevant operators introduce significant deviations $\Delta G_{\text {irr }}$ from the solid line. As $\rho W$ grows, however, the model Hamiltonian sinks deeper into the Kondo regime, and the agreement with the solid lines improves.

\section{B. Conductance for $U<D$}

We now consider the more realistic Coulomb repulsion $U=0.05 D$, which pushes the Kondo regime to the temperature range $k_{B} T \ll \min \left(\left|\varepsilon_{d}\right|, \varepsilon_{d}+U\right)$. As in Sec. VIII, we consider wire gate potentials, in the interval $0 \leqslant \rho W \leqslant 1$. To generate Kondo temperatures comparable to those in Fig. 7(b), for each wire potential we choose a dot-level width $\Gamma$ so that Eq. (9) yields the effective width $\Gamma_{w}=2 \times 10^{-3} D$ and let the dot energy run from $\varepsilon_{d}+U / 2=-0.04 D$ to $\varepsilon_{d}+U / 2=0.04 D$. Ampler than the scope of Figs. 7 and 8, this range provides an encompassing view of the Kondo and mixed-valence regimes.

\section{Conductance landscape}

Figure 13 shows the conductance as a function of the temperature and dot energy for $\rho W=0$. Analogous to Fig. $7(\mathrm{a})$, the plot is symmetric about the $\varepsilon_{d}+U / 2=0$ plane. In contrast with Fig. 7(a), however, the landscape displays a ridge, parallel to the $\varepsilon_{d}$ axis, at high temperatures. The ridge marks the crossover from the free-orbital fixed point, ${ }^{2}$ the temperature range associated with thermal energies above the charge-excitation energies $\Delta_{0}^{*} \approx\left|\varepsilon_{d}\right|$ and $\Delta_{2}^{*} \approx \varepsilon_{d}+U$, and the local-moment fixed point. Since the dot-level spectral density $\rho_{d}(\epsilon, T)$ peaks at $\Delta_{0}^{*}$ and $\Delta_{2}^{*},{ }^{46,52}$ the conductance first
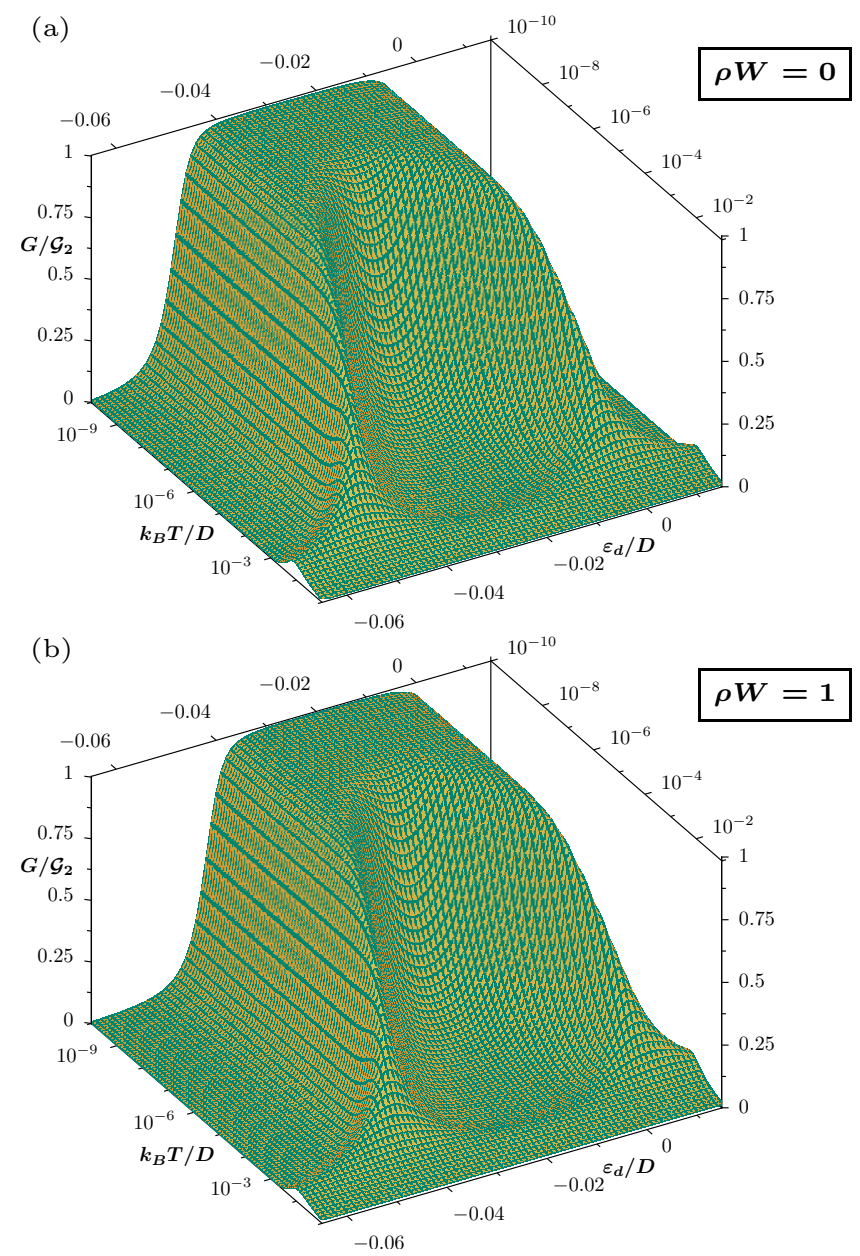

FIG. 13. (Color online) Conductance as a function of the temperature and dot energy for $U=0.05 D, \Gamma=0.002 D$, and wire gate potentials $\rho W=0$ (a) and $\rho W=1$ (b). In each case, a ridge separates the high-temperature range $k_{B} T \gtrsim U$ from the Kondo regime. In the Kondo regime, plots (a) and (b) reproduce the landscapes displayed in Figs. 7 and 8, respectively.

rises and then decays as the model Hamiltonian crosses over from the free-orbital fixed point to the Kondo regime.

The Kondo regime occupies the broad low-temperature sector of the plot where the excitation energies $\Delta_{0}^{*}$ and $\Delta_{2}^{*}$ exceed the dot-level width $\Gamma$ and the thermal energy $k_{B} T$. As the model Hamiltonian enters the Kondo regime, Eq. (49) takes control of the conductance and reproduces the gradual rise to the Kondo plateau depicted in Fig. 7(a).

At the outskirts of the Kondo regime, the narrow strip satisfying the inequality $\left|\Delta_{0}^{*}\right|<\Gamma\left(\left|\Delta_{2}^{*}\right|<\Gamma\right)$ defines the mixed-valence regime. As $\varepsilon_{d}+U / 2\left[-\left(\varepsilon_{d}+U / 2\right)\right]$ grows at fixed temperature, the conductance drops sharply in this regime. Beyond the mixed-valence strip, $\Delta_{0}^{*}\left(\Delta_{2}^{*}\right)$ is negative, $\left|\Delta_{0}^{*}\right|\left(\left|\Delta_{2}^{*}\right|\right)$ exceeds $\Gamma$, and the dot occupation $n_{d}$ approaches 0 (2). The dot magnetic moment vanishes, and the Coulomb blockade obstructs conduction even at $T=0$. In the entire temperature range $\left|\Delta_{0}^{*}\right| \geqslant k_{B} T\left(\left|\Delta_{2}^{*}\right| \geqslant k_{B} T\right)$, the conductance is close to zero.

Figure 13(b) shows the conductance as a function of the dot energy and temperature for $\rho W=1$. Comparison with Fig. 


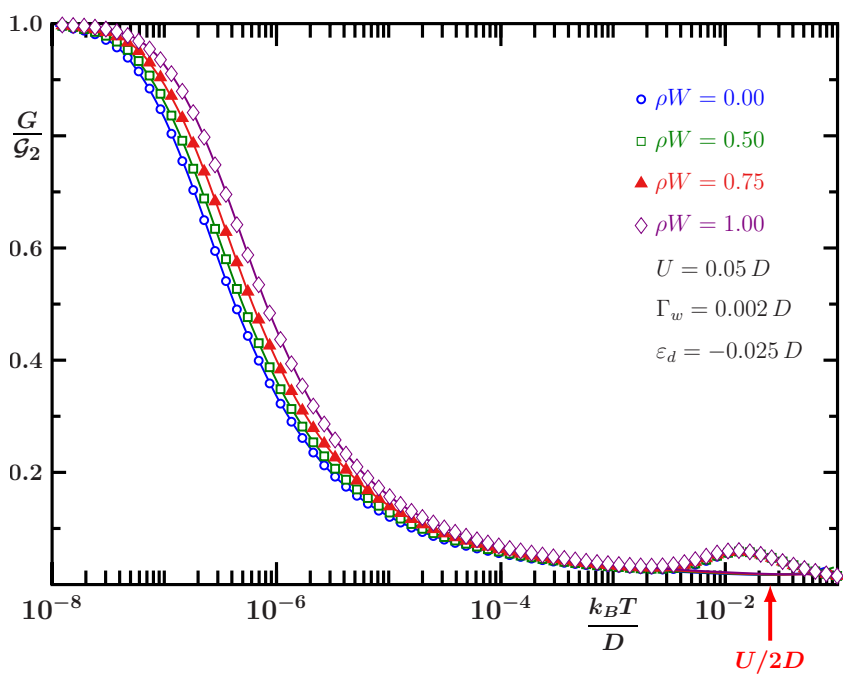

FIG. 14. (Color online) Temperature dependence of the conductance for the displayed model parameters. The vertical arrow points to thermal energy needed to add an electron to or remove an electron from the dot level. Each solid line represents Eq. (49) for the Kondo temperature defined by the equality $G\left(T=T_{K}\right)=\mathcal{G}_{2} / 2$ and the phase shift $\delta$ extracted from the low-energy spectrum of the model Hamiltonian.

13(a) shows that the wire potential moves the Kondo plateau and mixed-valence regimes to higher dot energies, a displacement analogous to the shift distinguishing Fig. 8(a) from Fig. 7(a). At high temperatures, near the free-orbital fixed point, only in the mixed-valence regime is the conductance significantly affected by the wire gate potential.

\section{Thermal dependence of the conductance}

Figure 14 displays the thermal dependence of the conductance for $\varepsilon_{d}+U / 2=0$ and four wire potentials, $\rho W=0,0.5$, 0.75 , and 1 . The symbols represent NRG results, while the solid lines represent Eq. (49) with the Kondo temperature $T_{K}$ extracted from the definition $G\left(T_{K}\right) \equiv \mathcal{G}_{2} / 2$ and the phase shift $\delta$ from the low-energy spectrum of the model Hamiltonian. In each case, the symbols agree impeccably with the solid line representing Eq. (49) in the range $k_{B} T<2$ $\times 10^{-3} D$. As indicated by the vertical arrow pointing to the lower horizontal axis, the $n_{d}=0$ and $n_{d}=2$ dot configurations become thermally accessible at higher temperatures. Charge excitations then control the dynamics of transport and make it insensitive to the wire potential. The circles, squares, triangles, and diamonds therefore depart from the solid lines to form a single, broad conductance peak, which corresponds to the high-temperature ridges in Fig. 13.

Figure 15 plots the conductance as a function of the temperature for $\varepsilon_{d}+U / 2=-0.015 D$ and five wire potentials: $\rho W=0,0.25,0.50,0.75$, and 1 . The charge excitation energies $\left|\varepsilon_{d}\right|$ and $\varepsilon_{d}+U$ are now different; the vertical arrow in the figure points to the lowest one and indicates proximity to the mixed-valence regime. The symbols are NRG data, while the solid lines depict Eq. (49). The open circles and filled squares agree very well with the solid line at temperatures below $k_{B} T=10^{-3} \mathrm{D}$ and draw a broad nonuniversal maximum near the indicated excitation energy.

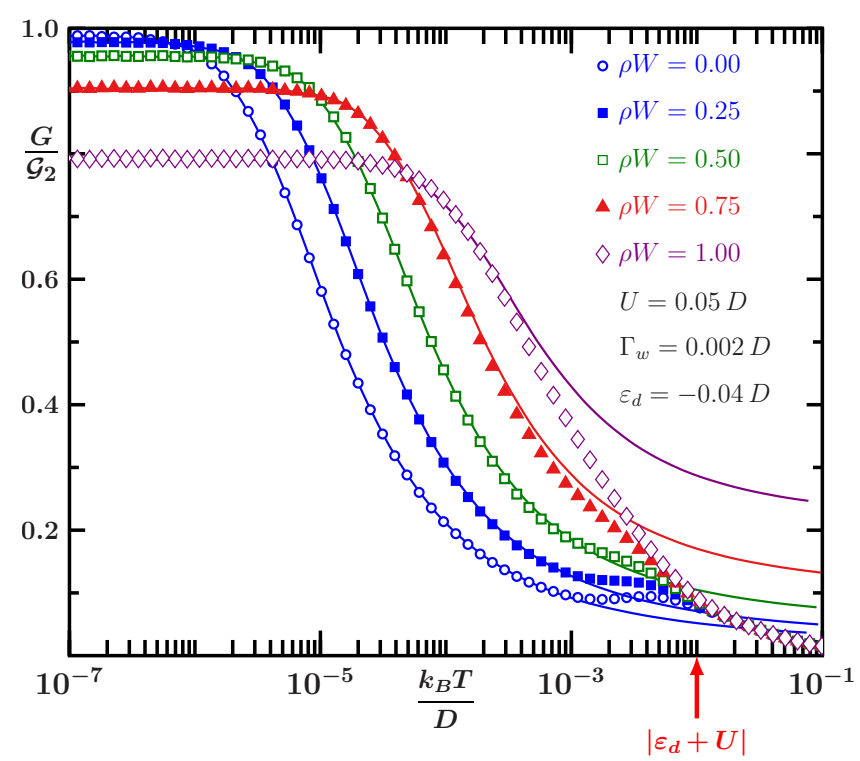

FIG. 15. (Color online) Temperature dependence of the conductance for the displayed model parameters. The vertical arrow points to thermal energy needed to add a second electron to the dot level, smaller than the energy necessary to remove the dot electron. As in Fig. 14, each solid line represents Eq. (49).

The circles and open squares in Fig. 15 follow the pattern set by Fig. 14. For $\rho W \geqslant 0.5$, however, the high-temperature peak in Fig. 15 first turns into an inflexion point and then disappears as the wire potential rises. A brief glance at Fig. 13 is sufficient to see that the dot energy $\varepsilon_{d}=-0.04 D$ moves from the Kondo to the mixed-valence regime as the wire potential grows from $\rho W=0$ to $\rho W=1$.

The filled triangles and open diamonds in Fig. 15 are in the mixed-valence regime. In the same way that the triangles and diamonds in Fig. 11 lie significantly below the pertinent solid lines, only above $\mathcal{G}_{2} / 2$ do the triangles and diamonds in Fig. 15 agree with Eq. (49), i.e., only below the Kondo temperature. This reinforces our finding that besides accurate at all temperatures in the Kondo regime, Eq. (49) is reliable at low temperatures even in the mixed-valence regime.

\section{Discussion}

In the Kondo regime, Eqs. (22a) and (22b) fix the highand the low-temperature conductances, respectively. Equation (49) shows that the universal function $G^{S}\left(T / T_{K}\right)$ controls the monotonic transition between the two limits. For $W=0$, in particular, the fixed-point values depend only on the ground-state phase shift $\delta$ and are symmetric with respect to $\mathcal{G}_{2} / 2: G_{L M}=\mathcal{G}_{2} \cos ^{2} \delta$ and $G_{F L}=\mathcal{G}_{2} \sin ^{2} \delta$. Thus, depending on $\delta$, the transition from $G_{L M}$ to $G_{F L}$ can be steeper or flatter. Since $\delta$ can never depart much from $\pi / 2$ in the Kondo regime, the argument of the trigonometric function on the right-hand side of Eq. (49) can never depart substantially from $\pi$, and as indicated by the two curves in Fig. 4, $G\left(T / T_{K}\right) \approx G^{S}\left(T / T_{K}\right) \pm 20 \%$. By contrast with this crude estimate, the mapping (49) gives excellent agreement with the symbols in the Kondo-regime curves in Fig. 9-12, 14, and 15 . 
The wire potential $W$ narrows the dot level and displaces the ground-state phase shift. Depending on the sign and magnitude of $W$, the phase shift can take any value in its domain of definition $-\pi / 2 \leqslant \delta \leqslant \pi / 2$. In the Kondo regime, the Friedel sum rule nonetheless prevents the difference $\delta-\delta_{W}$ from straying away from $\pi / 2$. All effects considered, the scattering potential $W$ displaces the conductance curve toward the symmetric limit $G\left(T / T_{K}\right)=G^{S}\left(T / T_{K}\right)$.

These findings are in line with the experimentally established notion that in the Kondo regime, SET conductances always decay with temperature..$^{5,14,36,38}$ This behavior contrasting with that of the conductance in the side-coupled geometry, ${ }^{31,32}$ we digress briefly to compare the two arrangements. As demonstrated in Ref. 30, a linear mapping analogous to Eq. (49) can be established between the side-coupled conductance and $G^{S}\left(T / T_{K}\right)$. Since the coefficient relating the two functions is $\cos (2 \delta)$, instead of $\cos \left[2\left(\delta-\delta_{w}\right)\right]$, the approximate equality $\delta-\delta_{w} \approx \pi / 2$ makes the coefficient sensitive to changes in $\delta_{w}$. Under a sufficiently strong wire potential, the sign of the coefficient can be reversed. Thus, the thermal dependence of the conductance through the sidecoupled device is tunable: ${ }^{35}$ a wire potential can turn a monotonically increasing function into a monotonically decreasing one. The embedded geometry of Fig. 1 is much less sensitive to $W$

In Fig. 1, the charge induced under the symmetric electrodes by the potential $W$ is $\pi$ times $\delta_{w}$. According to the Friedel sum rule, ${ }^{42}$ the difference $\delta-\delta_{W}$ is the charge of the Kondo cloud, the additional charge that piles up at the wire tips surrounding the dot as the temperature is lowered past $T_{K}$. Neutrality makes the charge of the Kondo cloud equal to the dot occupancy. Since the symmetric condition $n_{d}=1$ maximizes the low-temperature conductance, one expects $G(T=0)$ to be ballistic for $2\left(\delta-\delta_{W}\right)=\pi$, a conclusion in agreement with Eq. (49). Since the screening charge is always nearly unitary, one expects the low-temperature conductance to be close to the conductance quantum, in agreement with the plots in Figs. 9-12, 14, and 15.

\section{Prospect of comparison with experiment}

Equation (49) is analogous to the expression relating the universal function $G^{S}$ to the conductance in the side-coupled arrangement. ${ }^{30}$ Both expressions are linear, and the linearity simplifies the comparison with experimental data. To fit the conductances $G_{i}(i=1, \ldots, N)$ measured at $N$ temperatures $T_{i}$, we follow the procedure illustrated by Fig. 3 in Ref. 30. We start with a trial Kondo temperature $T_{K}$ and compute the scaled temperatures $\tau_{i}=T_{i} / T_{K}(i=1, \ldots, N)$. Since we know the universal function $G^{S}\left(T / T_{K}\right)$, it is then a simple matter to compute the set $G^{S}\left(\tau_{i}\right)(i=1, \ldots, N)$ of universal conductances at the scaled temperatures. According to Eq. (49), plotted as a function of $G^{S}\left(\tau_{i}\right), G_{i}$ should lie on a straight line. If the line is crooked, we have to choose a new trial $T_{K}$ and repeat the procedure. In practice, we seek the Kondo temperature optimizing the least-squares linear fit to the plot of $G_{i}$ vs $G^{S}\left(\tau_{i}\right)$.

In addition to yielding the Kondo temperature, the linear regression determines the argument $2\left(\delta-\delta_{W}\right)$ of the trigono- metric function on the right-hand side of Eq. (49). Convenient as the SET therefore is to measure the ground-state phase shift $\delta$, its interferometric scope is narrow, for the phase shifts in the embedded geometry are always close to $\delta_{W}+\pi / 2$. By comparison, the side-coupled device constitutes an interferometer of more practical value because it is free from this limitation. ${ }^{30,53}$

\section{SUMMARY}

The first part of this report derived our central result, Eq. (49), which maps the conductance in the embedded geometry onto the universal conductance for the symmetric Anderson model (11) linearly. As our discussion of the result showed, while the Kondo temperature sets the temperature scale, the dot charge controls the mapping, even with a gate potential applied to the wires. If the dot occupation $n_{d}$ is unitary, the mapping reduces to the equality $G\left(T / T_{K}\right)=G^{S}\left(T / T_{K}\right)$, the result found by Costi et al. ${ }^{29}$ Elsewhere in the Kondo regime, the dot occupation being still close to unity $G\left(T / T_{K}\right)$ is never qualitatively different from $G^{S}\left(T / T_{K}\right)$. Relative deviations as large as $20 \%$ may nevertheless separate the two functions at low temperatures.

By contrast with the approximation $G\left(T / T_{K}\right) \approx G^{S}\left(T / T_{K}\right)$, Eq. (49) describes the conductance exactly in the Kondo regime and is hence the appropriate instrument to describe experimental or numerical data. In particular, once fitted to an experimental curve, the mapping determines the Kondo temperature $T_{K}$, as well as the dot charge.

The essentially exact numerical results in the second part of the paper presented an overview of zero-bias conduction through a quantum dot embedded in the conduction path of a nanodevice. Equation (49), a mapping explicitly parametrized by the Kondo temperature and dot occupancy, guided our discussion of the computed conduction curves in the Kondo regime. We also surveyed the neighboring domains of the parametrical space: the free-orbital and mixed-valence regimes. In the former, instead of scaling with $T / T_{K}$, the thermal dependence of the conductance scales with $k_{B} T / E_{c}$, where $E_{c}$ is a charge-excitation energy, $E_{c}=\min \left(\Delta_{0}^{*}, \Delta_{2}^{*}\right)$. In the mixed-valence regime, the effective dot-level width $\Gamma_{w}$ setting the temperature scale, the mapping (49) becomes asymptotically exact for $k_{B} T \ll \Gamma_{w}$ and only witnesses the final rise of the conductance to its low-temperature limit $G(T$ $=0)=\mathcal{G}_{2} \sin ^{2}\left(\delta-\delta_{w}\right)$.

By contrast, in the Kondo regime the mapping is asymptotically exact for $k_{B} T \ll \min D, \Delta_{0}^{*}, \Delta_{2}^{*}$ and hence describes accurately the rise of the conductance throughout the Kondo crossover. The distinction between the Kondo and the mixedvalence regimes is a problem often encountered in the laboratory. 5,32 The mapping to the universal curve offers a solution of practical value.

\section{ACKNOWLEDGMENTS}

This work was supported by the CNPq and FAPESP.

\section{APPENDIX A: PROPERTIES OF THE FIXED-POINT HAMILTONIANS}

\section{Diagonalization}

The LM and FL are described by conduction-band Hamiltonians of the form 


$$
H^{*}=\sum_{k} \epsilon_{k} a_{k}^{\dagger} a_{k}+W^{*} f_{0}^{\dagger} f_{0} .
$$

We want to bring $H^{*}$ to the diagonal form

$$
H^{*}=\sum_{\ell} \varepsilon_{\ell} g_{\ell}^{\dagger} g_{\ell},
$$

where

$$
g_{\ell}=\sum_{q} \alpha_{\ell q} a_{q} .
$$

To this end, we compare the expressions for the commutator $\left[g_{\ell}, H^{*}\right]$ obtained from Eqs. (A1) and (A2), from which it follows that

$$
\alpha_{\ell q}=\frac{1}{\varepsilon_{\ell}-\epsilon_{q}} \frac{W^{*}}{N} \sum_{k} \alpha_{\ell k} .
$$

Summation of both sides over $q$ then leads to the eigenvalue condition,

$$
1=\frac{W^{*}}{N} \sum_{q} \frac{1}{\varepsilon_{\ell}-\epsilon_{q}} .
$$

Inspection of this equality shows that, with exception of a split-off energy, which makes $\mathcal{O}(1 / N)$ contributions to the low-energy properties, the $\varepsilon_{\ell}$ are shifted by less than $\Delta$ from the $\epsilon_{k}$. We therefore refer to the closest conduction energy $\epsilon_{\ell}$ to label each eigenvalue and define its phase shift $\delta_{\ell}$ with the expression

$$
\varepsilon_{\ell} \equiv \epsilon_{\ell}-\frac{\Delta}{\pi} \delta_{\ell}
$$

This definition substituted for $\varepsilon_{\ell}$, a Sommerfeld-Watson transformation ${ }^{54}$ evaluates the sum on the right-hand side of Eq. (A5),

$$
\frac{1}{N} \sum_{q} \frac{1}{\varepsilon_{\ell}-\epsilon_{q}}=-\pi \rho \cot \delta_{\ell}+\rho \int_{-D}^{D} \frac{1}{\varepsilon_{\ell}-\epsilon} d \epsilon .
$$

The eigenvalue Eq. (A5) therefore determines the phase shift,

$$
\cot \delta_{\ell}=-\frac{1}{\pi \rho W^{*}}+\frac{1}{\pi} \int_{-D}^{D} \frac{1}{\varepsilon_{\ell}-\epsilon} d \epsilon .
$$

At low energies, the contribution of the last term on the right-hand side, of $\mathcal{O}(\epsilon / D)$, can be neglected, and the phase shift becomes uniform,

$$
\tan \delta=-\pi \rho W^{*} .
$$

Next, we square both sides of Eq. (A4) and sum the result over $q$ to determine the coefficients $\alpha_{\ell q}$.

$$
\sum_{q} \alpha_{\ell q}^{2}=\left(\sum_{k} \alpha_{\ell k} \frac{W^{*}}{N}\right)^{2} \sum_{q} \frac{1}{\left(\varepsilon_{\ell}-\epsilon_{q}\right)^{2}} .
$$

The sum on the left-hand side is unitary. To evaluate the sum over $q$ on the right-hand side, we differentiate Eq. (A7) with respect to $\epsilon_{\ell}$. With relative error $\mathcal{O}(1 / N)$, we find that

$$
\frac{1}{N^{2}} \sum_{q} \frac{1}{\left(\varepsilon_{\ell}-\epsilon_{q}\right)^{2}}=\left(\frac{\pi \rho}{\sin \delta_{\ell}}\right)^{2} .
$$

Thus, Eq. (A10) reduces to

$$
W^{*} \sum_{k} \alpha_{\ell k}=-\frac{1}{\pi \rho} \sin \delta_{\ell},
$$

the negative sign insuring that $\alpha_{k k} \rightarrow 1$ for $W^{*} \rightarrow 0$. From Eq. (A4) it then follows that

$$
\alpha_{\ell q}=\frac{\Delta}{\epsilon_{q}-\varepsilon_{\ell}} \frac{\sin \delta_{\ell}}{\pi} \quad\left(\varepsilon_{\ell} \ll D\right) .
$$

\section{Energy moments of the matrix elements of the eigenoperators $g_{\ell}$}

This appendix shows that, given two eigenstates $|m\rangle$ and $|n\rangle$ of the Hamiltonian $H_{A}$, and considered the operators $f_{0}$, $f_{1}, \phi_{0}$, and $\phi_{1}$ defined by Eqs. (6), (30), (26), and (27), respectively, the matrix element $\left\langle m\left|f_{0}\right| n\right\rangle\left(\left\langle m\left|f_{1}\right| n\right\rangle\right)$ is a linear combination of the matrix elements $\left\langle m\left|\phi_{0}\right| n\right\rangle$ and $\left\langle m\left|\phi_{1}\right| n\right\rangle$ with coefficients that are independent of the eigenstates $|m\rangle$ and $|n\rangle$, as long their eigenvalues $E_{m}$ and $E_{n}$, respectively, are much smaller than the conduction bandwidth. We define the dimensionless energy $\mathcal{E}_{m n} \equiv\left(E_{m}-E_{n}\right) / D$ and assume that

$$
\left|\mathcal{E}_{m n}\right| \ll 1 \text {. }
$$

Under this assumption, we consider the energy moments

$$
M_{m n}^{(p)} \equiv \frac{1}{\sqrt{N}} \sum_{\ell}\left(\frac{\varepsilon_{\ell}}{D}\right)^{p}\left\langle m\left|g_{\ell}\right| n\right\rangle \quad(p=0,1, \ldots),
$$

where $g_{\ell}\left(\epsilon_{\ell}\right)$ is one of the eigenstates (eigenvalues) defined by Eq. (A2).

Since the $M_{m n}^{p}$ are universal, to evaluate them it is sufficient to consider the symmetric Hamiltonian (11), for which the phase shift $\delta_{L M}=0$ so that $g_{k}, \varepsilon_{k}, \phi_{0}$, and $\phi_{1}$ coincide with $a_{k}, \epsilon_{k}, f_{0}$, and $f_{1}$, respectively.

From Eq. (11), we then have that

$$
\left[g_{\ell}, H_{A}^{S}\right]=\varepsilon_{\ell} g_{\ell}+\frac{V}{\sqrt{N}} c_{d} .
$$

Multiplication of both sides by $\left(\varepsilon_{\ell} / D\right)^{p-1}$ followed by summation over $\ell$ leads to the coupled recursive relations,

$$
\begin{gathered}
M_{m n}^{(p)}=-\mathcal{E}_{m n} M_{m n}^{(p-1)}-\frac{V}{p}\left\langle m\left|c_{d}\right| n\right\rangle \quad(p=1,3, \ldots), \\
M_{m n}^{(p)}=-\mathcal{E}_{m n} M_{m n}^{(p-1)} \quad(p=2,4, \ldots) .
\end{gathered}
$$

Reduced to a matrix equation, this system is easily solved,

$$
\begin{gathered}
M_{m n}^{(p)}=-\frac{V}{p}\left\langle m\left|c_{d}\right| n\right\rangle\left(1+\sum_{r=1}^{p-2} \frac{\left(\mathcal{E}_{m n}\right)^{r}}{r}\right)+M_{m n}^{(0)}\left(\mathcal{E}_{m n}\right)^{p} \\
(p=1,3, \ldots)
\end{gathered}
$$

and 


$$
M_{m n}^{(p)}=-\frac{V}{p}\left\langle m\left|c_{d}\right| n\right\rangle \sum_{r=1}^{p-1} \frac{\left(\mathcal{E}_{m n}\right)^{r}}{r}+M_{m n}^{(0)}\left(\mathcal{E}_{m n}\right)^{p} \quad(p=2,4, \ldots),
$$

where the primed sums are restricted to odd $r$ 's.

In view of Eq. (A14), the terms proportional to $\left(\mathcal{E}_{m n}\right)^{p}(p$ $\geqslant 1)$ on the right-hand sides of these two equalities can be neglected. From Eq. (A17) we then see that $M_{m n}^{(1)}=$ $-V\left\langle m\left|c_{d}\right| n\right\rangle$ and all the other odd moments are proportional to $M_{m n}^{(1)}$, and from Eq. (A18), we see that the only nonzero even moment is $M_{m n}^{(0)}$. More specifically,

$$
M_{m n}^{(p)}=\left\{\begin{array}{cc}
\frac{\left\langle m\left|\phi_{1}\right| n\right\rangle}{p} & (p=1,3, \ldots) \\
0 & (p=2,4, \ldots) .
\end{array}\right.
$$

This simple relation suggests that we define the following orthonormal basis of conduction states:

$$
\phi_{\ell} \equiv \sqrt{\frac{2 \ell+1}{N}} \sum_{p} P_{\ell}\left(\epsilon_{p}\right) g_{p} \quad(\ell=0,1, \ldots),
$$

where $P_{\ell}$ denotes a Legendre polynomial. In particular, with $\ell=0(\ell=1)$, we recover Eq. (26) [Eq. (27)].

Given two eigenstates $|m\rangle$ and $|n\rangle$ of $H_{A}$, the matrix element $\left\langle m\left|\phi_{\ell}\right| n\right\rangle$ is a linear combination of the moments $M_{m n}^{(r)}$ $(r=1,3, \ldots, \ell$ for odd $\ell$, or $r=2,4, \ldots, \ell$ for even $p)$. It follows from Eq. (A19), then that $\left\langle m\left|\phi_{\ell}\right| n\right\rangle \sim\left\langle m\left|\phi_{1}\right| n\right\rangle$ ( $\ell$ $=3,5, \ldots)$, while $\left\langle m\left|\phi_{\ell}\right| n\right\rangle=0(\ell=2,4, \ldots)$. More generally, the matrix element of any conduction operator is a linear combination of $\left\langle m\left|\phi_{0}\right| n\right\rangle$ and $\left\langle m\left|\phi_{1}\right| n\right\rangle$. In particular,

$$
\left\langle m\left|f_{i}\right| n\right\rangle=\sum_{j=0}^{1} \alpha_{i j}\left\langle m\left|\phi_{j}\right| n\right\rangle \quad(i=0,1),
$$

where $f_{0}$ and $f_{1}$ are the operators defined by Eqs. (6) and (30), respectively, and $\alpha_{i j}(i, j=0,1)$ are model-parameter dependent constants.

\section{APPENDIX B: FIXED-POINT CONDUCTANCES}

This appendix derives an expression for the spectral density $\rho_{d}(\epsilon, T)$ at the fixed points. We start with Eq. (28), from which we obtain an expression for the matrix element of the conduction operator $a_{q}^{\dagger}$ between two low-energy eigenstates $|m\rangle$ and $|n\rangle$ of $H_{A}$,

$$
\begin{aligned}
\left\langle m\left|a_{q}^{\dagger}\right| n\right\rangle= & \frac{1}{\sqrt{N}} \frac{V}{E_{m}-E_{n}-\epsilon_{q}}\left\langle m\left|c_{d}^{\dagger}\right| n\right\rangle \\
& +\frac{W}{N} \frac{1}{E_{m}-E_{n}-\epsilon_{q}}\left\langle m\left|\sum_{p} a_{p}^{\dagger}\right| n\right\rangle .
\end{aligned}
$$

Summation of both sides over $q$ leads to an expression for the matrix element in the last term on the right-hand side,

$$
\left\langle m\left|\sum_{p} a_{p}^{\dagger}\right| n\right\rangle\left(1-W \mathcal{S}_{m n}\right)=\sqrt{N} V\left\langle m\left|c_{d}^{\dagger}\right| n\right\rangle \mathcal{S}_{m n},
$$

where

$$
\mathcal{S}_{m n} \equiv \frac{1}{N} \sum_{q} \frac{1}{E_{m}-E_{n}-\epsilon_{q}},
$$

which brings Eq. (B1) to the form

$$
\left\langle m\left|a_{q}^{\dagger}\right| n\right\rangle=\frac{\left\langle m\left|c_{d}^{\dagger}\right| n\right\rangle}{\sqrt{N}\left(E_{m}-E_{n}-\epsilon_{q}\right)} \frac{V}{1-W \mathcal{S}_{m n}} .
$$

Consider now this equality at one of the two fixed points, LM or FL. The fixed-point Hamiltonian has then the quadratic form (A2), which defines the complete basis of the operators $g_{\ell}$. The matrix element $\left\langle m\left|g_{\ell}^{\dagger}\right| n\right\rangle$ vanishes unless $|m\rangle=g_{\ell}^{\dagger}|n\rangle$, which implies $E_{m}=E_{n}+\varepsilon_{\ell}$. At a fixed point, therefore, the sum on the right-hand side of Eq. (B3) reduces to that in Eq. (A7), i.e.,

$$
\mathcal{S}_{m n}=-\pi \rho \cot \delta_{*},
$$

where we have ignored the last term on the right-hand side of Eq. (A7) because at a fixed point the ratio $\varepsilon_{\ell} / D \rightarrow 0$. Equation (B5) suggests that we introduce the phase shift $\delta_{W}$, defined by

$$
\tan \delta_{W} \equiv-\pi \rho W,
$$

to simplify Eq. (B4),

$$
\left\langle m\left|a_{q}^{\dagger}\right| n\right\rangle=\frac{V\left\langle m\left|c_{d}^{\dagger}\right| n\right\rangle}{\sqrt{N}\left(E_{m}-E_{n}-\epsilon_{q}\right)} \frac{\sin \delta_{*} \cos \delta_{W}}{\sin \left(\delta_{*}-\delta_{W}\right)} .
$$

In analogy with Eq. (A3) we can, moreover, write

$$
g_{\ell}=\gamma_{\ell 0} c_{d}+\sum_{q} \gamma_{\ell q} a_{q},
$$

with normalized coefficients,

$$
\gamma_{\ell 0}^{2}+\sum_{q} \gamma_{\ell q}^{2}=1
$$

Equation (B8) is easily inverted to yield the expressions

$$
a_{q}=\sum_{\ell} \gamma_{\ell q} g_{\ell}
$$

and

$$
c_{d}=\sum_{\ell} \gamma_{\ell 0} g_{\ell}
$$

Substitution of Eq. (B10) for $a_{q}$ on the left-hand side of Eq. (B7) and of Eq. (B11) for $c_{d}$ on the right-hand side then yields

$$
\gamma_{\ell q}^{2}=\frac{V^{2} \gamma_{\ell 0}^{2}}{N\left(\varepsilon_{\ell}-\epsilon_{q}\right)^{2}}\left(\frac{\sin \delta_{*} \cos \delta_{W}}{\sin \left(\delta_{*}-\delta_{W}\right)}\right)^{2} .
$$

We divide both sides by $N$, sum them over $q$, and substitute Eq. (A11) for the resulting sum on the right-hand side to find that

$$
\sum_{q} \gamma_{\ell q}^{2}=N V^{2} \gamma_{\ell 0}^{2}\left(\frac{\pi \rho \cos \delta_{W}}{\sin \left(\delta_{*}-\delta_{W}\right)}\right)^{2} .
$$


Substitution in the second term on the left-hand side of Eq. (B9) now shows that, with error $\mathcal{O}(1 / N)$, the fixed-point matrix elements $\left\langle m\left|c_{d}^{\dagger}\right| n\right\rangle$ are constants, dependent only on the phase shift and scattering potential,

$$
\left|\left\langle m\left|c_{d}^{\dagger}\right| n\right\rangle\right|^{2}=\frac{1}{N V^{2}} \frac{\sin ^{2}\left(\delta_{*}-\delta_{W}\right)}{\pi^{2} \rho^{2} \cos ^{2} \delta_{W}} .
$$

The fixed-point spectral density, as one would also expect, is temperature independent,

$$
\rho_{d}(\epsilon, T)=\frac{1}{N V^{2} \mathcal{Z}} \sum_{m, n} e^{-\beta E_{m}} \frac{\sin ^{2}\left(\delta_{*}-\delta_{W}\right)}{\pi^{2} \rho^{2} \cos ^{2} \delta_{W}} \delta\left(\epsilon_{\ell}-\epsilon\right),
$$

equivalent to

$$
\rho_{d}(\epsilon)=\frac{\sin ^{2}\left(\delta_{*}-\delta_{W}\right)}{\pi \Gamma \cos ^{2} \delta_{W}} .
$$

With $W=0$, we recover the celebrated expression ${ }^{42}$

$$
\rho_{d}(\epsilon)=\frac{\sin ^{2} \delta_{*}}{\pi \Gamma} .
$$

More generally, however, to obtain the fixed-point spectral densities, we set $\delta^{*}=\delta$ at the FL, and $\delta^{*}=\delta-\pi / 2$ at the LM, from which it results that

$$
\begin{gathered}
\rho_{d}^{L M}=\frac{\cos ^{2}\left(\delta-\delta_{W}\right)}{\pi \Gamma_{w}}, \\
\rho_{d}^{F L}=\frac{\sin ^{2}\left(\delta-\delta_{W}\right)}{\pi \Gamma_{w}} .
\end{gathered}
$$

Substitution of Eqs. (B18a) and (B18b) for $\rho_{d}$ on the righthand side of Eq. (8) leads to Eqs. (22a) and (22b), respectively.

\section{APPENDIX C: ZERO-BIAS CONDUCTANCE}

By contrast with the coupling to the impurity, which is independent of the odd operators $b_{k}$ defined by Eq. (4b), the Hamiltonian describing a bias voltage couples to $b_{k}$ 's. Preliminary to the discussion of the conductance, it is therefore convenient to derive results for $b_{k}$ 's analogous to those in Appendix A. Specifically, given the formal equivalence between Eqs. (7) and (A1), we can follow the steps in that Appendix to write $H_{B}$ in the diagonal form

$$
H_{B}=\sum \widetilde{\varepsilon}_{\ell} \tilde{g}_{\ell}^{\dagger} \widetilde{g}_{\ell},
$$

with

$$
\widetilde{g}_{\ell}=\sum_{k} \widetilde{\alpha}_{\ell, k} b_{k}
$$

and derive a result analogous to Eq (A12). At low energies, in particular, i.e., for $\left|\widetilde{\varepsilon}_{\ell}\right| \ll D$, the eigenvalues $\widetilde{\varepsilon}_{\ell}$ are uniformly spaced, with the phase shift $\delta_{w}$ defined by Eq. (B6), and

$$
\sum_{k} \widetilde{\alpha}_{\ell, k}=\cos \delta_{W}
$$

Multiplication of both sides by $\tilde{g}_{\ell}$ and summation over $\ell$ then shows that

$$
\sum_{k}\left\langle\tilde{m}\left|b_{k}\right| \widetilde{n}\right\rangle=\cos \delta_{w} \sum_{\ell}\left\langle\tilde{m}\left|\tilde{g}_{\ell}\right| \tilde{n}\right\rangle
$$

for any pair $|\tilde{m}\rangle,|\tilde{n}\rangle$ of low-energy eigenstates of $H_{B}$.

It is likewise convenient to compute the following commutator:

$$
\left[H, a_{k}^{\dagger} b_{k}\right]=\frac{V}{\sqrt{N}} c_{d}^{\dagger} b_{k}+\frac{W}{N} \sum_{q}\left(a_{q}^{\dagger} b_{k}-a_{k}^{\dagger} b_{q}\right),
$$

from which we see that, given two eigenstates $\left|\Psi_{m}\right\rangle$ and $\left|\Psi_{n}\right\rangle$ of $H_{A}$ with eigenvalues $E_{m}$ and $E_{n}$, respectively,

$$
\begin{aligned}
\left\langle\Psi_{m}\left|a_{k}^{\dagger} b_{k}\right| \Psi_{n}\right\rangle= & \frac{V}{\sqrt{N}} \frac{\left\langle\Psi_{m}\left|c_{d}^{\dagger} b_{k}\right| \Psi_{n}\right\rangle}{E_{m}-E_{n}} \\
& +\frac{W}{N} \sum_{q} \frac{\left\langle\Psi_{m}\left|a_{q}^{\dagger} b_{k}-a_{k}^{\dagger} b_{q}\right| \Psi_{n}\right\rangle}{E_{m}-E_{n}} .
\end{aligned}
$$

\section{Current}

To calculate the conductance, we can, for instance, examine the current flowing into the $R$ wire,

$$
\hat{I}=\frac{d q_{R}}{d t}=-\frac{i e}{\hbar}\left[H, \sum_{k} c_{k R}^{\dagger} c_{k R}\right]
$$

i.e.,

$$
\hat{I}=-\frac{i e}{2 \hbar}\left[H, \sum_{k}\left(a_{k}^{\dagger} a_{k}+b_{k}^{\dagger} b_{k}-\left(a_{k}^{\dagger} b_{k}+\text { H.c. }\right)\right)\right],
$$

which reduces to

$$
\hat{I}=\frac{i e}{2 \hbar} \frac{V}{\sqrt{N}} c_{d}^{\dagger} \sum_{k}\left(a_{k}+b_{k}\right)+\text { H.c. }
$$

because summed over $k$, the last term on the right-hand side of Eq. (C5) vanishes.

\section{Conductance}

To induce a current, we add to the model Hamiltonian an infinitesimal slowly growing perturbation that lowers the chemical potential of the $R$ wire relative to that of the $L$ wire,

$$
H_{\mu} \equiv \Delta \mu h_{\mu}(t)=-e \frac{\Delta \mu}{2} \sum_{k}\left(c_{k R}^{\dagger} c_{k R}-c_{k L}^{\dagger} c_{k L}\right) e^{\eta t / \hbar},
$$

with an infinitesimal shift $\Delta \mu$.

Projected on the basis of $a_{k}$ 's and $b_{k}$ 's, $h_{\mu}$ reads

$$
h_{\mu}(t)=-\frac{e}{2} \sum_{k}\left(a_{k}^{\dagger} b_{k}+\text { H.c. }\right) e^{\eta t / \hbar},
$$

and Eq. (C6) shows that 


$$
\left\langle\Psi_{m}\left|h_{\mu}(t)\right| \Psi_{n}\right\rangle=-\frac{e V}{2 \sqrt{N}} e^{\eta t / \hbar} \sum_{k} \frac{\left\langle\Psi_{m}\left|c_{d}^{\dagger} b_{k}-b_{k}^{\dagger} c_{d}\right| \Psi_{n}\right\rangle}{E_{m}-E_{n}} .
$$
tance,

Standard linear response theory relates $h_{\mu}$ to the conduc-

$$
G(T)=-\frac{i}{\mathcal{Z} \hbar} \int_{-\infty}^{0} \sum_{m} e^{-\beta E_{m}}\left\langle\Psi_{m}\left|\left[\hat{I}, h_{\mu}(t)\right]\right| \Psi_{m}\right\rangle d t
$$

where $\mathcal{Z}$ is the partition function at the temperature $T$.

Comparison with Eq. (C11) shows that the operators $a_{k}$ within the parentheses on the right-hand side of that equality make no contribution to the conductance. We therefore define

$$
\hat{I}_{b} \equiv \frac{i e}{2 \hbar} \frac{V}{\sqrt{N}} c_{d}^{\dagger} \sum_{k} b_{k}+\text { H.c. }
$$

and rewrite Eq. (C13),

$$
G(T)=-\frac{i}{\mathcal{Z} \hbar} \int_{-\infty}^{0} \sum_{m} e^{-\beta E_{m}}\left\langle\Psi_{m}\left|\left[\hat{I}_{b}, h_{\mu}(t)\right]\right| \Psi_{m}\right\rangle d t .
$$

Following the insertion of a completeness sum $\Sigma_{n}|n\rangle\langle n|$ on the right-hand side of Eq. (C15), straightforward manipulations lead to the familiar expression,

$$
G(T)=\frac{1}{\mathcal{Z}} \sum_{m, n}\left(e^{-\beta E_{m}}-e^{-\beta E_{n}}\right) \frac{\left\langle\Psi_{m}\left|\hat{I}_{b}\right| \Psi_{n}\right\rangle\left\langle\Psi_{n}\left|h_{\mu}(0)\right| \Psi_{m}\right\rangle}{E_{m}-E_{n}+i \eta} .
$$

On the right-hand side, we now substitute Eq. (C12) [Eq. (C14)] for $h_{\mu}\left(\hat{I}_{b}\right)$. This yields

$$
\begin{aligned}
G(T)= & -i \frac{e^{2}}{4 \hbar} \frac{V^{2}}{N \mathcal{Z}_{m, n, k, q}}\left(\frac{\left\langle\Psi_{m}\left|b_{q}^{\dagger} c_{d}\right| \Psi_{n}\right\rangle\left\langle\Psi_{n}\left|c_{d}^{\dagger} b_{k}\right| \Psi_{m}\right\rangle}{E_{m}-E_{n}+i \eta}\right. \\
& \left.+\frac{\left\langle\Psi_{m}\left|c_{d}^{\dagger} b_{k}\right| \Psi_{n}\right\rangle\left\langle\Psi_{n}\left|b_{q}^{\dagger} c_{d}\right| \Psi_{m}\right\rangle}{E_{m}-E_{n}+i \eta}\right) \frac{e^{-\beta E_{m}}-e^{-\beta E_{n}}}{E_{m}-E_{n}} .
\end{aligned}
$$

Aided by Eq. (C4), we can now trade the sum over the conduction operators $b_{k}$ for a sum over the eigenoperators $\tilde{g}_{\ell}$,

$$
\begin{aligned}
G(T)= & -i \frac{e^{2}}{2 h} \frac{\Gamma_{W}}{N \rho \mathcal{Z}} \sum_{m, n, \ell, \ell^{\prime}}\left(\frac{\left\langle\Psi_{m}\left|\widetilde{g}_{\ell^{\prime}}^{\dagger} c_{d}\right| \Psi_{n}\right\rangle\left\langle\Psi_{n}\left|c_{d}^{\dagger} \tilde{g}_{\ell}\right| \Psi_{m}\right\rangle}{E_{m}-E_{n}+i \eta}\right. \\
& \left.+\frac{\left\langle\Psi_{m}\left|c_{d}^{\dagger} \widetilde{g}_{\ell}\right| \Psi_{n}\right\rangle\left\langle\Psi_{n}\left|\widetilde{g}_{\ell^{\prime}}^{\dagger} c_{d}\right| \Psi_{m}\right\rangle}{E_{m}-E_{n}+i \eta}\right) \frac{e^{-\beta E_{m}}-e^{-\beta E_{n}}}{E_{m}-E_{n}} .
\end{aligned}
$$

Since the $\widetilde{g}_{\ell}$ diagonalize $H_{B}$, only the terms with $\ell=\ell^{\prime}$ contribute to the sum on the right-hand side. We interchange the indices $m$ and $n$ in the second term within the parentheses on the right-hand side to show that

$$
G(T)=\frac{\pi e^{2}}{h} \frac{\beta \Gamma_{w}}{\rho N \mathcal{Z}} \sum_{m, n, \ell} e^{-\beta E_{m}}\left|\left\langle\Psi_{m}\left|c_{d}^{\dagger} \tilde{g}_{\ell}\right| \Psi_{n}\right\rangle\right|^{2} \delta\left(E_{m}-E_{n}\right) .
$$

Since $\left|\Psi_{m}\right\rangle=|m\rangle|\widetilde{m}\rangle$, where $|m\rangle(|\widetilde{m}\rangle)$ is an eigenstate of $H_{A}$ (of the quadratic Hamiltonian $H_{B}$ ), the right-hand side splits into two coupled sums,

$$
\begin{aligned}
G(T)= & \frac{\pi e^{2}}{h} \frac{\beta \Gamma_{W}}{N \rho \mathcal{Z}} \sum_{m, n, \ell} e^{-\beta E_{m}}\left|\left\langle m\left|V c_{d}^{\dagger}\right| n\right\rangle\right|^{2} \\
& \left.\times \delta\left(E_{m}-E_{n}-\tilde{\epsilon}_{\ell}\right) \sum_{\tilde{m}, \tilde{n}} e^{-\beta E_{\tilde{m}}\langle\widetilde{m}}\left|\widetilde{g}_{\ell}\right| \widetilde{n}\right\rangle\left\langle\widetilde{n}\left|\widetilde{g}_{\ell}^{\dagger}\right| \widetilde{m}\right\rangle .
\end{aligned}
$$

The second sum is equal to $\mathcal{Z}_{b}\left[1-f\left(\widetilde{\epsilon}_{p}\right)\right]$, where $f(\epsilon)$ is the Fermi function and $\mathcal{Z}_{b}$ is the partition function for the Hamiltonian $H_{b}$. The identity

$$
-\frac{1}{f(\epsilon)} \frac{\partial f}{\partial \epsilon}=\beta(1-f(\epsilon))
$$

then turns Eq. (C19) into

$$
\begin{aligned}
G(T)= & \frac{e^{2}}{h \mathcal{Z}_{a}} \frac{\pi \Gamma_{W}}{\rho N} \sum_{m, n, \ell} \frac{e^{-\beta E_{m}}}{f\left(\tilde{\boldsymbol{\epsilon}}_{\ell}\right)}\left(-\frac{\partial f}{\partial \boldsymbol{\epsilon}}\right)_{\tilde{\varepsilon}_{\ell}}\left|\left\langle m\left|c_{d}^{\dagger}\right| n\right\rangle\right|^{2} \\
& \times \delta\left(E_{m}-E_{n}-\tilde{\boldsymbol{\epsilon}}_{\ell}\right),
\end{aligned}
$$

where $\mathcal{Z}_{a}$ is the partition function for the Hamiltonian $H_{A}$.

Definition (10) of the spectral density $\rho_{d}(\epsilon, T)$ allows us to rewrite Eq. (C21) as

$$
G(T)=\frac{e^{2}}{h} \frac{\pi \Gamma_{W}}{\rho N} \sum_{\ell}\left(-\frac{\partial f}{\partial \epsilon}\right)_{\tilde{\varepsilon}_{\ell}} \rho_{d}\left(\widetilde{\epsilon}_{\ell}\right),
$$

from which Eq. (8) follows.

\footnotetext{
*Present address: Instituto de Física, Universidade Federal Fluminense, Niterói 24210-346, RJ, Brazil.

${ }^{1}$ P. W. Anderson, Phys. Rev. 124, 41 (1961).

${ }^{2}$ H. R. Krishna-murthy, J. W. Wilkins, and K. G. Wilson, Phys. Rev. B 21, 1003 (1980).

${ }^{3}$ N. Andrei, K. Furuya, and J. H. Lowenstein, Rev. Mod. Phys. 55, 331 (1983).
}

${ }^{4}$ A. M. Tsvelick and P. B. Wiegmann, Adv. Phys. 32, 453 (1983).

${ }^{5}$ D. Goldhaber-Gordon, J. Göres, M. A. Kastner, H. Shtrikman, D. Mahalu, and U. Meirav, Phys. Rev. Lett. 81, 5225 (1998).

${ }^{6} \mathrm{~J}$. W. Wilkins, in Valence Instabilities, edited by P. Wachter and H. Boppart (North-Holland, New York, 1982), p. 1.

${ }^{7}$ C. L. Lin, A. Wallash, J. E. Crow, T. Mihalisin, and P. Schlottmann, Phys. Rev. Lett. 58, 1232 (1987). 
${ }^{8}$ L. I. Glazman and M. E. Raikh, JETP Lett. 47, 452 (1987).

${ }^{9}$ T. K. Ng and P. A. Lee, Phys. Rev. Lett. 61, 1768 (1988).

${ }^{10} \mathrm{~S}$. Hershfield, J. H. Davies, and J. W. Wilkins, Phys. Rev. Lett. 67, 3720 (1991).

${ }^{11}$ Y. Meir and N. S. Wingreen, Phys. Rev. Lett. 68, 2512 (1992).

${ }^{12}$ N. S. Wingreen and Y. Meir, Phys. Rev. B 49, 11040 (1994).

${ }^{13}$ V. Madhavan, W. Chen, T. Jamneala, M. F. Crommie, and N. S. Wingreen, Science 280, 567 (1998).

${ }^{14}$ W. G. van der Wiel, S. D. Francheschi, T. Fujisawa, J. M. Elzerman, S. Tarucha, and L. P. Kouwenhoven, Science 289, 2105 (2000).

${ }^{15}$ W. Hofstetter, J. König, and H. Schoeller, Phys. Rev. Lett. 87, 156803 (2001).

${ }^{16}$ W. G. van der Wiel, S. De Franceschi, J. M. Elzerman, T. Fujisawa, S. Tarucha, and L. P. Kouwenhoven, Rev. Mod. Phys. 75, 1 (2003).

${ }^{17}$ N. Agrait, A. L. Yeyati, and J. M. van Ruitenbeek, Phys. Rep. 377, 81 (2003).

${ }^{18}$ S. Kirchner, L. J. Zhu, Q. M. Si, and D. Natelson, Proc. Natl. Acad. Sci. U.S.A. 102, 18824 (2005).

${ }^{19}$ M. F. Crommie, Science 309, 1501 (2005).

${ }^{20}$ R. Franco, M. S. Figueira, and E. V. Anda, Phys. Rev. B 67, 155301 (2003).

${ }^{21}$ C. Romeike, M. R. Wegewijs, W. Hofstetter, and H. Schoeller, Phys. Rev. Lett. 96, 196601 (2006).

${ }^{22}$ L. G. G. V. Dias da Silva, N. P. Sandler, K. Ingersent, and S. E. Ulloa, Phys. Rev. Lett. 97, 096603 (2006).

${ }^{23}$ R. Zitko and J. Bonca, Phys. Rev. B 73, 035332 (2006).

${ }^{24}$ L. G. G. V. Dias da Silva, K. Ingersent, N. Sandler, and S. E. Ulloa, Phys. Rev. B 78, 153304 (2008).

${ }^{25}$ M. Grobis, I. G. Rau, R. M. Potok, H. Shtrikman, and D. Goldhaber-Gordon, Phys. Rev. Lett. 100, 246601 (2008).

${ }^{26}$ Y. Oreg and D. Goldhaber-Gordon, Phys. Rev. Lett. 90, 136602 (2003).

${ }^{27}$ Y.-S. Fu et al., Phys. Rev. Lett. 99, 256601 (2007).

${ }^{28}$ R. Bulla, T. A. Costi, and T. Pruschke, Rev. Mod. Phys. 80, 395 (2008).

${ }^{29}$ T. Costi, A. Hewson, and V. Zlatic, J. Phys.: Condens. Matter 6, 2519 (1994).

${ }^{30}$ A. C. Seridonio, M. Yoshida, and L. N. Oliveira, Europhys. Lett. 86, 67006 (2009).

${ }^{31}$ K. Kobayashi, H. Aikawa, A. Sano, S. Katsumoto, and Y. Iye, Phys. Rev. B 70, 035319 (2004).

${ }^{32}$ M. Sato, H. Aikawa, K. Kobayashi, S. Katsumoto, and Y. Iye, Phys. Rev. Lett. 95, 066801 (2005).

${ }^{33}$ S. Katsumoto, M. Sato, H. Aikawa, and Y. Iye, Physica E (Amsterdam) 34, 36 (2006).
${ }^{34}$ T. Otsuka, E. Abe, S. Katsumoto, Y. Iye, G. L. Khym, and K. Kang, J. Phys. Soc. Jpn. 76, 084706 (2007).

${ }^{35}$ S. Katsumoto, J. Phys.: Condens. Matter 19, 233201 (2007).

${ }^{36}$ D. Goldhaber-Gordon, H. Shtrikman, D. Mahalu, D. AbuschMagder, U. Meirav, and M. A. Kastner, Nature (London) 391, 156 (1998).

${ }^{37}$ J. Göres, D. Goldhaber-Gordon, S. Heemeyer, M. A. Kastner, H. Shtrikman, D. Mahalu, and U. Meirav, Phys. Rev. B 62, 2188 (2000).

${ }^{38}$ W. Liang, M. P. Shores, M. Bockrath, and J. R. Long, Nature (London) 417, 725 (2002).

${ }^{39}$ L. H. Yu, Z. K. Keane, J. W. Ciszek, L. Cheng, M. P. Stewart, J. M. Tour, and D. Natelson, Phys. Rev. Lett. 93, 266802 (2004).

${ }^{40}$ L. H. Yu, Z. K. Keane, J. W. Ciszek, L. Cheng, J. M. Tour, T. Baruah, M. R. Pederson, and D. Natelson, Phys. Rev. Lett. 95, 256803 (2005).

${ }^{41}$ J. R. Schrieffer and P. A. Wolff, Phys. Rev. 149, 491 (1966).

${ }^{42}$ D. C. Langreth, Phys. Rev. 150, 516 (1966).

${ }^{43} \mathrm{~A}$ sum of irrelevant terms, of which $\Sigma_{k} \epsilon_{k} c_{k \mu}^{\dagger} \boldsymbol{\sigma}_{\mu \nu} \phi_{0 \nu} S$ and $\Sigma_{k} \epsilon_{k} c_{k \mu}^{\dagger} \boldsymbol{\sigma}_{\mu \nu} \Sigma_{k} \epsilon_{k} c_{k \nu} \boldsymbol{S}$ are examples, has been neglected on the right-hand side of Eq. (25). While they affect the Kondo temperature and the ground-state phase shift, the irrelevant terms have no effect upon the universal properties. This approximation is thoroughly discussed in Refs. 2 and 45.

${ }^{44}$ K. G. Wilson, Rev. Mod. Phys. 47, 773 (1975).

${ }^{45}$ H. R. Krishna-murthy, J. W. Wilkins, and K. G. Wilson, Phys. Rev. B 21, 1044 (1980).

${ }^{46}$ A. C. Hewson, The Kondo Problem to Heavy Fermions (Cambridge University Press, Cambridge, 1993).

${ }^{47}$ M. Yoshida, M. A. Whitaker, and L. N. Oliveira, Phys. Rev. B 41, 9403 (1990).

${ }^{48}$ L. Komzsik, The Lanczos Method: Evolution and Application (SIAM, Philadelphia, 2003).

${ }^{49}$ V. L. Campo and L. N. Oliveira, Phys. Rev. B 72, 104432 (2005).

${ }^{50}$ W. C. Oliveira and L. N. Oliveira, Phys. Rev. B 49, 11986 (1994).

${ }^{51}$ F. D. M. Haldane, Phys. Rev. Lett. 40, 416 (1978).

${ }^{52}$ H. O. Frota and L. N. Oliveira, Phys. Rev. B 33, 7871 (1986).

${ }^{53}$ A. C. Seridonio, M. Yoshida, and L. N. Oliveira, following paper, Phys. Rev. B 80, 235318 (2009).

${ }^{54}$ J. Matthews and R. L. Walker, Mathematical Methods of Physics (Addison-Wesley, New York, 1971).

${ }^{55}$ Reference 45 discusses the mixed-valence regime under the heading "transitional cases" in Sec. III E.

${ }^{56}$ With $\varepsilon_{d}=-4 D$ and $\rho W=0.75$, although in the mixed-valence regime, the model Hamiltonian is close to the Kondo regime. 